\title{
Schulformen und sozioökonomischer Erfolg im jungen Erwachsenenalter: Werden unterschiedliche Ausbildungswege auf dem Arbeitsmarkt gleich honoriert?
}

\author{
Michael Becker $\mathbb{D} \cdot$ Julia Tetzner $\cdot$ Jürgen Baumert
}

Eingegangen: 6. August 2019 / Überarbeitet: 4. Mai 2020 / Angenommen: 21. September 2020 / Online publiziert: 12 . Oktober 2020

(C) Der/die Autor(en) 2020

Zusammenfassung Inwiefern der Besuch unterschiedlicher Schulformen Lernen und Bildungsverläufe beeinflusst, ist ein vieldiskutiertes Thema. Arbeiten zu Effekten der Schulformen für den Erfolg auf dem Arbeitsmarkt liegen jedoch bislang nur wenige vor. Hier setzt der vorliegende Beitrag an, in dem in einer längsschnittlichen Perspektive ( $N=5292$ Personen) überprüft wird, welche Effekte der Besuch unterschiedlicher Schulformen in der Sekundarstufe (vom Beginn der 7. Klasse) auf spätere berufliche Merkmale (Berufsprestige, Einkommen und Arbeitslosigkeit im Alter von knapp 31 Jahren) aufweist. Die Ergebnisse weisen prima facie auf Unterschiede zwischen Schulformen hin, aber ein Großteil der Effekte lässt sich durch schulische bzw. berufliche Abschlüsse, sowie individuelle und soziale Hintergrundunterschiede erklären. Zumindest für das Einkommen und Arbeitslosigkeit erscheinen vor allem die schulischen und beruflichen Abschlüsse maßgebend. Der Effekt des Besuchs der Hauptschule im Vergleich zu anderen Schulformen bleibt jedoch mit einem niedrigeren Berufsprestige assoziiert, auch unter zusätzlicher Kontrolle des individuellen und sozialen Hintergrundes. Die Ergebnisse legen nahe, dass unterschiedliche Wege durch die Schulformen der Sekundarstufe auf dem Arbeitsmarkt partiell differenzi-

Zusatzmaterial online Zusätzliche Informationen sind in der Online-Version dieses Artikels (https:// doi.org/10.1007/s11618-020-00973-7) enthalten.

Prof. Dr. M. Becker $(\bowtie)$

Institut für Schulentwicklungsforschung (IFS), TU Dortmund,

CDI-Campus Nord, Vogelpothsweg 78, 44227 Dortmund, Deutschland

E-Mail: becker@dipf.de

Dr. J. Tetzner

DIPF | Leibniz-Institut für Bildungsforschung und Bildungsinformation,

Rostocker Straße 6, 60323 Frankfurt a.M., Deutschland

E-Mail: tetzner@dipf.de

Prof. Dr. J. Baumert

Max-Planck-Institut für Bildungsforschung, Lentzeallee 94, 14195 Berlin, Deutschland

E-Mail: sekbaumert@mpib-berlin.mpg.de 
ell entlohnt werden: nicht für das Einkommen und Erwerbslosigkeit, aber für den beruflichen Status.

Schlüsselwörter Schulformen der Sekundarstufe · Abschlusszertifikate · Berufliches Prestige · Einkommen · Arbeitslosigkeit · Längsschnittliche Studie

\title{
School types and socioeconomic success in young adulthood: are different educational pathways equally rewarded on the labor market?
}

\begin{abstract}
There has been much discussion of the extent to which the secondary school type attended influences learning and educational trajectories in Germany. However, little is yet known about the effects of school type on later labor market outcomes. This article aims to fill this gap by taking a longitudinal perspective on the effects of attending different secondary school types (following $N=5292$ persons from the beginning of grade 7) on later occupational characteristics (job prestige, income, and unemployment at age 31 years). At first glance, the results point to differences between school types, but these effects are to a large extent explained by school/vocational qualifications and by individual and social differences. When it comes to predicting income and unemployment, at least, vocational qualifications, in particular, seem to be decisive. The effect of attending a vocational-track Hauptschule relative to the other school types remains associated with lower occupational status, however, even with additional control for individual and social background. The findings suggest that different educational pathways in the form of different secondary school types are indeed to some extent differently rewarded on the labor market: not in terms of income and unemployment, but in terms of occupational status.
\end{abstract}

Keywords Income - Longitudinal study - Occupational status - School type · School-leaving certificates · Unemployment

\section{Einleitung}

Einer der nachhaltigsten Trends der letzten Jahrzehnte ist die tiefgreifende Veränderung der Bildungsbeteiligung in der Sekundarstufe, die sogenannte Bildungsexpansion: Weiterführende Bildung wird in Deutschland (wie auch weltweit) immer stärker nachgefragt (Baumert et al. 2019; Müller 1998; Wolter 1989). Hand in Hand mit dieser Entwicklung zeichnete sich auch eine Entkoppelung zwischen besuchter Schulform und Abschlusszertifikat ab, so dass sich immer mehr Personen für ,,alternative" Bildungswege entscheiden und Zertifikate, wie der mittlere Abschluss bzw. das Abitur, zu substantiellen Teilen nicht in den traditionellen Schulformen wie der Realschule bzw. dem Gymnasium erworben werden (vgl. auch Köller et al. 2004a; Trautwein et al. 2008).

Weitgehend ungeklärt ist hierbei, welche Bedeutung unterschiedliche in der Sekundarstufe besuchte Schulformen für die sozioökonomische Stellung wie den beruflichen Status, Einkommen oder Arbeitslosigkeit im Erwachsenenalter haben. So 
wird gerade der Hauptschule eine ungünstige Wirkung nachgesagt. In dieser Diskussion wird jedoch häufig nicht expliziert, ob eigentlich über Schülerinnen und Schüler an Hauptschulen gesprochen wird oder ob Schülerinnen und Schüler mit einem Hauptschulabschluss gemeint sind: Mutmaßliche Effekte von Hauptschulen könnten somit nicht (nur) aus der spezifischen Lernumwelt resultieren, sondern (eher) Effekte des erworbenen Zertifikats sein - unabhängig davon, ob dieser Abschluss nun an einer Hauptschule oder einer anderen Schule erworben wurde. Ähnlich lässt sich auch für den Erwerb des Abiturs an alternativen Schulformen fragen, inwiefern dieser Abschluss äquivalent zu einem Abitur ist, das an einem traditionellen Gymnasium erworben wurde. Schuchart und Schimke (2019) haben jüngst erst auf die Diskrepanz zwischen der Bedeutung dieses Themas, vor allem in Hinblick auf die Verteilungsgerechtigkeit des deutschen Schulsystems und dem gleichzeitig bestehenden Mangel an Studien zu dieser Frage, hingewiesen. In der Tat existieren bislang kaum Arbeiten, die die Bedeutung von Schulformen vs. Zertifikaten in Hinblick auf den späteren sozioökonomischen Erfolg auf dem Arbeitsmarkt untersuchen (siehe aber Fend 2014; Schuchart und Schimke 2019; Sterrenberg 2014).

Ziel des vorliegenden Beitrages ist es, zur Schließung dieser Forschungslücke beizutragen und zu untersuchen, welche Bedeutung der Schulform einerseits und den Abschlusszertifikaten andererseits für späteren sozioökonomischen Erfolg zukommen. Die Studie untersucht dies, nach unserem Kenntnisstand erstmalig, systematisch für alle Schulformen und Abschlusszertifikate in einer längsschnittlichen Analyse, um so die Effekte von Schulform, Qualifikation und Abschlusszertifikat für den Erfolg auf dem Arbeitsmarkt (beruflicher Status, Einkommen, Arbeitslosigkeit) explizit voneinander zu trennen. Die Studie setzt mit dem Übergang in die 7. Klasse ein und ermöglicht es somit, individuelle Voraussetzungen und familiäre Hintergrundfaktoren zu berücksichtigen und ein Gesamtbild der zentralen strukturellen und individuellen Einflussfaktoren zu zeichnen.

\section{Forschungslage}

\subsection{Bildungsexpansion und Entkopplung von Schulform und Abschlusszertifikat}

Betrachtet man die Entwicklung des allgemeinbildenden Schulsystems in Deutschland (und auch weltweit), so lässt sich unverkennbar ein Trend hin zu mehr und weiterführender Bildung identifizieren. Für das deutsche Schulsystem lässt sich dies anhand der alten Bundesländer hervorragend erläutern: In den 1950er-Jahren besuchten knapp $80 \%$ eines Jahrgangs die Volks- und Hauptschulen, und nur jeweils rund $10 \%$ ein Gymnasium. 50 Jahre später, zu Beginn der 2000er-Jahre besuchten lediglich rund $20 \%$ eine Hauptschule, aber mehr als $30 \%$ ein Gymnasium. Realschulen sowie Gesamtschulen und Schulen mit mehreren Bildungsgängen teilten sich die verbleibende Schülerschaft ungefähr paritätisch (Baumert et al. 2008). Dieser Trend hat sich in den jüngsten Jahren weiter fortgesetzt, so dass das Gymnasium mittlerweile die am stärksten besuchte Schulform ist und sich somit zur neuen „Haupt“Schule wandelte (vgl. auch Autorengruppe Bildungsberichterstattung 2018). 
Diese Entwicklung ging mit einer Flexibilisierung von Anschlussmöglichkeiten einher, was maßgeblich auf Reformbemühungen der 1970er-Jahre zurückzuführen ist. Im Zuge der damaligen Neuerungen wurden curriculare Änderungen im Sekundarschulsystem vorgenommen, so dass prinzipiell weiterhin Abstufungen zwischen den Schulformen vorgesehen waren, die aber eher quantitativer als qualitativer Art sein sollten (Becker et al. 2006; Leschinsky 2003, 2008). Hauptschulen sollten z. B. nicht mehr (allein) praktisch qualifizieren, und so wurde auch dort unter anderem Englisch als obligatorisches Unterrichtsfach eingeführt, um eine bessere Anschlussfähigkeit an die anderen Schulformen zu gewährleisten.

In der Tat wurden vertikale Anschlussmöglichkeiten ausgeweitet und gleichzeitig verstärkt nachgefragt. So bemühte man sich seit den Schulstrukturdebatten in den 1970er-Jahren, alternative Schulformen mit allgemeinbildenden Oberstufen anzubieten und damit alternative Wege zur allgemeinbildenden Hochschulreife zu ebnen (vgl. Brauckmann und Neumann 2004). Dies findet sich etwa durch die Einführung von Gesamtschulen umgesetzt (Köller et al. 2004a). Auch die Reformbemühungen in jüngeren Jahren richteten sich insbesondere darauf, Schulformen der Mittelstufe mit mehr oder weniger direkten vertikalen Anschlussoptionen zu versehen (Baumert et al. 2017; Becker et al. 2016). In der jüngsten Strukturreform in Berlin wurde beispielsweise das 4-gliedrige Schulsystem formal abgeschafft und in ein 2-WegeSystem überführt, das nur noch das Gymnasium und eine sogenannte Integrierte Sekundarschule vorhält (Maaz et al. 2013; Neumann et al. 2017b).

In diesem Zusammenhang wird auch diskutiert, inwiefern die vertikale Öffnung dazu beiträgt, sozial bedingte Ungleichheiten bei der Teilhabe am Besuch weiterführender Schulformen zu verringern. Aus der Übergangsforschung ist bekannt, dass beispielsweise der Übergang von der Grundschule in die weiterführende Schule zwar zum Großteil leistungsbasiert erfolgt, daneben aber auch sozial bedingte (direkte) Effekte im Übergangsverhalten zu finden sind (Ditton 2010; Dumont et al. 2014; Maaz et al. 2008; Watermann et al. 2014; Weiss 2013; Baumert et al. 2019). Die Flexibilisierung der Abschlüsse innerhalb der Schulformen und der vertikalen Anschlussoptionen könnte dazu beitragen, mögliche negative Konsequenzen der Entscheidungen an früheren Übergangsschwellen abzumildern. Ob dies zutrifft, ist durchaus umstritten. Die alternativen Wege scheinen in jedem Fall den zentralen Pfad darzustellen, auf dem Kinder aus weniger privilegierten und bildungsfernen Familien den Zugang zur Hochschulreife erwerben (Buchholz und Pratter 2017; Trautwein et al. 2011). Gleichzeitig finden sich aber auch Hinweise darauf, dass gerade Personen privilegierterer sozialer Herkunft die vertikalen Anschlussoptionen stärker nutzen als Personen aus weniger privilegierten Verhältnissen (vgl. Buchholz und Pratter 2017; Hillmert 2014; Hillmert und Jacob 2005). Eine Frage, die sich hieran anschließt, wird in der Literatur unter dem Konzept der Effectively-Maintained-Inequality (Lucas 2001, 2009; Schindler 2014) diskutiert. Dies meint, dass sich im Zuge der Bildungsexpansion unterschiedliche soziale Gruppen in Hinblick auf Bildungsabschlüsse (d.h. Bildungsquantität) zunehmend ähnlicher werden. Entsprechend könnten sich Distinktionsprozesse in andere Bildungsdimensionen, vor allem die der Bildungsqualität verlagern. Wenn sich z. B. durch den Erwerb des Abiturs selbst keine oder nur geringe Unterschiede begründen lassen, weil der Anteil in 
allen Bevölkerungsgruppen hoch ist, könnten sich Unterschiede darin ausdrücken, an welcher Schulform das Abitur erworben wird.

Es lässt sich zusammenfassend feststellen, dass ein breiter Trend hin zu weiterführender Allgemeinbildung zu verzeichnen ist. Dies schlägt sich nicht nur in einer stärkeren Nachfrage nach weiterführenden Schulformen, und hier vor allem einer stärkeren Nachfrage nach Gymnasien nieder, sondern geht auch damit einher, dass weiterführende Qualifikationen vermehrt auf nicht-traditionellen Bildungswegen, wie z. B. an Gesamtschulen oder gymnasialen Oberstufen in beruflichen Schulen, erworben werden. Dies macht insbesondere die Frage virulent, inwiefern es langfristig für den sozioökonomischen Erfolg von Bedeutung ist, welche Schulform eine Person in der Sekundarstufe besuchte oder ob lediglich die Abschlusszertifikate und die tatsächlich erworbenen Qualifikationen erfolgsrelevant sind. Auch aus Perspektive der Verteilungsgerechtigkeit im deutschen Schulsystem erscheint diese Frage bedeutsam.

\subsection{Effekte von Schulformen oder von schulischen Qualifikationen?}

Ob Schulformen eine diskriminierende Wirkung entfalten, wurde aus unterschiedlichen Perspektiven wiederholt debattiert. Zum einen wurde auf einer psychologischen Ebene die Frage nach der erlebten sozialen Identität je nach besuchter Schulform diskutiert. Insbesondere dem Besuch geringer qualifizierender Schulformen, d.h. Hauptschulen (aber auch Sonder- und Förderschulen), hafte ein negatives Stigma an bzw. fördere die Entwicklung ungünstiger sozialer Identitäten (vgl. z. B. Knigge und Hannover 2011; Solga und Wagner 2001). Dies könnte sich unter anderem ungünstig auf die Leistungsbereitschaft in der Schule, die Ausbildungsaspirationen und die Übergänge in die Berufswelt auswirken. Auch im internationalen Diskurs wird die Frage der negativen sozialen Identität und daraus resultierender negativer Einstellungen zu Bildung gering Qualifizierter erörtert (z. B. Kuppens et al. 2017). Entsprechend könnte ein Effekt unterschiedlicher schulischer Milieus darin bestehen, dass durch spezifische psychologische Sozialisationsprozesse (in den Hauptschulen im Wesentlichen negativ, in den Gymnasien positiv) ein längerfristiger direkter Effekt über die Zeit in der Schule hinausgeht, vor allem wenn sich die mutmaßlich geringere Leistungsbereitschaft beispielsweise im Bewerbungsverhalten fortschreibt.

Unabhängig davon, was der Hauptschule im Negativen und umgekehrt dem Gymnasium im Positiven zugeschrieben wird: Was häufig in diesem Diskurs offen bleibt, ist die Frage, ob von den Schulformen selbst oder von den entsprechenden Abschlusszertifikaten gesprochen wird. Zwar sind die klassischen Schulformen in der Tat primär mit den stereotyp zugeschriebenen Abschlusszertifikaten assoziiert: Auch zum heutigen Zeitpunkt, zu dem die Korrelation zwischen besuchter Schulform und Abschlusszertifikat weniger stark ist, werden die meisten Hauptschulabschlüsse an Hauptschulen erworben (zumindest in den Bundesländern, in denen Hauptschulen noch existieren) und umgekehrt die meisten Hochschulzugangsberechtigungen an Gymnasien (Köller et al. 2004a). Aber die Zugänge zu Abschlusszertifikaten sind nicht einzelnen Schulformen exklusiv vorbehalten.

Eine Arbeit, die zumindest von der psychologischen Seite her die Bedeutung der Differenzierung von Schulformen und Abschlüssen verdeutlichen konnte, wurde 
jüngst von Dumont et al. (2017) vorgelegt: Die Autorinnen und Autoren untersuchten, inwiefern die Wahrnehmung der eigenen Kompetenz und der persönlichen Chancen auf dem Arbeitsmarkt, sowie das schulische Engagement, entweder mit der Schulform, der Komposition der Lerngruppe oder dem individuell erwarteten $\mathrm{Ab}$ schlusszertifikat assoziiert waren. Die Autorinnen und Autoren machten sich mit einer Berliner Stichprobe die Entkoppelung von Schulabschluss und Schulform zunutze und konnten für Schülerinnen und Schüler an den nicht-gymnasialen Schulformen zeigen, dass gerade die Wahrnehmung der eigenen Chancen auf dem Arbeitsmarkt an das Abschlusszertifikat und nicht die Schulform gekoppelt war: Schülerinnen und Schüler mit erwartetem Hauptschulabschluss schätzten sich allesamt ungünstiger ein als solche mit Realschulabschluss, und zwar unabhängig davon, ob diese Schülerinnen und Schüler eine Haupt-, Real- oder Gesamtschule besuchten. Diese psychologische Wahrnehmung der eigenen Chancen ist selbstverständlich nicht mit den Mechanismen gleichzusetzen, die auf dem Arbeitsmarkt tatsächlich von Bedeutung für den beruflichen sozioökonomischen Erfolg im Erwachsenenalter sind. Die Arbeit unterstreicht aber die Notwendigkeit, diese Aspekte zu differenzieren.

\subsection{Schulformeffekte auf den sozioökonomischen Erfolg}

Aus einer humankapitaltheoretischen Perspektive lässt sich postulieren, dass von Seiten des Arbeitsmarktes die Bildung von Humankapital entscheidend ist, wozu neben beruflicher Erfahrung vor allem die Schule beiträgt. In der Literatur ist breit belegt, dass mehr Bildung, in der Regel gemessen in Bildung in Jahren, mit größerem sozioökonomischem Erfolg verbunden ist (Heckman et al. 2006; Psacharopoulos und Patrinos 2004). Analog zur psychologischen Selbstwahrnehmung, wie sie bei Dumont et al. (2017) untersucht wurde, spräche dies dafür, dass vor allem den Abschlusszertifikaten und weniger den besuchten Schulformen eine Wirkung zukommt. Andererseits ist vielfach berichtet worden, dass die unterschiedlichen Schulformen mit unterschiedlichen Leistungsniveaus einhergehen, die nicht selektionsbedingt sind, sondern partiell auch durch schulformabhängige Entwicklungsmilieus erzeugt werden können (Köller et al. 2004a, b; Trautwein et al. 2010).

Langfristig könnte dies auch für den sozioökonomischen Erfolg auf dem Arbeitsmarkt relevant werden, wenngleich bislang unklar ist, inwiefern dies zumindest im deutschen System durch unterschiedliche berufliche Ausbildungsverläufe vermittelt wird. Grundlegend ist der deutsche Arbeitsmarkt stark segmentiert und der Zugang reguliert, was nicht nur universitäre Berufe (z. B. Zugang zu Berufen wie Ärztinnen und Ärzten) betrifft, sondern auch im Unterschied v. a. zu angelsächsischen Ländern den nicht-akademischen Bereich umfasst. Auch dieser Bereich ist durch die dualen (betrieblichen) und die schulischen Berufsausbildungen stark (korporatistisch) strukturiert, was bis in vormoderne, zünftische Traditionen zurückreicht (vgl. Baethke 2008; Protsch 2014). Insofern kommt gerade der beruflichen Bildung ${ }^{1}$ eine gate keeping Funktion zu. Dabei sollten die beruflichen Qualifikationen in weiten Teilen

\footnotetext{
1 Analog zu Kleinert und Jacob (2012) verwenden wir im Folgenden, sofern nicht anders spezifiziert, den Begriff berufliche Bildung als Überbegriff für sowohl berufliche duale und vollzeitschulische Berufsausbildung als auch für die (fach-)hochschulische Abschlüsse.
} 
die vorgelagerten Qualifikationen und Ausbildungswege mediieren, unter anderem da der Zugang zu den Teilsegmenten wieder an allgemeinbildende Mindestqualifikationen gebunden ist (z. B. setzt ein Studium in der Regel ein Abitur voraus; siehe aber zu Studierenden ohne Abitur Ordemann 2019). Umgekehrt besteht jedoch die Möglichkeit einer Entscheidung ,nach unten“, beispielsweise mit Abitur eine Ausbildung zu wählen, die lediglich eine Hauptschul- oder mittlere Reife voraussetzt.

Einen anderen wichtigen Aspekt stellt das Zusammenspiel schulischer und beruflicher Bildung für die weitere Individualentwicklung auf dem Arbeitsmarkt dar. Die international vergleichende Forschung legte nahe, dass Schulsysteme mit expliziten tracking-Strukturen (wie in Deutschland) unter anderem mit einer stärkeren Leistungsheterogenität einhergehen und auch die Korrelation zwischen dem sozialen Hintergrund und der Leistung tendenziell stärker ausfällt (Bol und van de Werfhorst 2013; Chmielewski und Reardon 2016). Demgegenüber scheint aber, gewissermaßen kompensierend, zu stehen, dass in diesen Ländern gerade die berufliche Bildung besonders stark ist und diese in der Regel direkt an die Sekundarstufenstrukturen und damit einhergehende Zertifikate anschließt, was zum Beispiel den Übergang von der Schule in den Beruf in diesen gegliederten Systemen reibungsloser zu machen scheint. Spezifischer lässt sich dies unter anderem filtertheoretisch begründen (Spence 1973; Weiss 1995): Schulische und beruflich-institutionalisierte Ausbildung nimmt nicht nur für die Vermittlung von Kompetenzen eine besondere Stellung ein, sondern sie macht die (prinzipiell nicht direkt beobachtbaren) Qualifikationen durch Zeugnisse zumindest in Teilen erkenn- und interpretierbar. Im Platzierungsprozess auf dem Arbeitsmarkt stellen Zertifikate dann die Signale dar, die Arbeitgeber nutzen, um auf die (zunächst unbekannten) Kenntnisse und Qualifikationen, also auf die Eignung von Bewerberinnen und Bewerbern zu schließen und daraufhin die Selektion zu treffen (Spence 1973; Weiss 1995). Vor allem in Systemen wie dem deutschen, das in allen Bereichen sekundärer und postsekundärer Bildung durch ein stark strukturiertes und formalisiertes Ausbildungswesen charakterisiert ist, sind Zeugnisse sowohl für Arbeitnehmer*innen als auch Arbeitsgeber*innen generell verfügbare Qualifikationsindikatoren (anders als z. B. in Systemen wie den angelsächsischen ohne weitgehend formalisierte berufliche Bildung; vgl. Bol und van de Werfhorst 2013). Die stärkere Zertifizierung macht die Qualifikationen mutmaßlich einfacher kenntlich. Es stellt sich hierbei aber auch die Frage, ob und in welchem Ausmaß neben der beruflichen Bildung allgemeinbildende schulische Abschlusszertifikate und - im Sinne der hier interessierenden spezifischen Fragestellung - die besuchten Schulformen der Sekundarstufe direkt prädiktiv für soziökonomischen Erfolg sind. Aus filtertheoretischen Überlegungen heraus träte ein direkter Effekt der Schulformen etwa dann auf, wenn neben den eigentlichen beruflichen (oder schulischen) Qualifikationen auch die Institutionen, an denen Qualifikationen erworben wurden, als Signale in den Bewerbungsprozess einbezogen würden.

\subsection{Schulformeffekte auf sozioökonomischen Erfolg: Spezifischer Forschungsstand}

Zur Prüfung und Trennung dieser Effekte gibt es nach unserem Kenntnisstand bislang nur die LiFE-Studie, die hierüber unter Berücksichtigung unterschiedlicher 
sozialer und kognitiver Hintergrundvariablen längsschnittlich Auskunft gibt. Fend (2009a, b, 2014; Georg 2009) untersuchte aus einer systemischen Perspektive, inwiefern gegliederte oder ungegliederte Schulsysteme zu unterschiedlichen sozioökonomischen Erträgen führen. Hierzu machte er sich die LiFE-Daten mit ihren quasi-experimentellen Variationen in der regionalen Einführung von Gesamtschulen zunutze. Zwar zeigten sich in der Sekundarstufe Effekte, die auf ein besseres meritokratisches Funktionieren in ungegliederten Gesamtschulsystemen hinwiesen (d.h. eine stärkere Abhängigkeit von kognitiven und eine geringere Abhängigkeit von sozialen Faktoren). Im Erwachsenenalter fanden sich jedoch kaum Hinweise darauf, dass ein Gesamtschulsystem zu anderen sozioökonomischen Erträgen führte als das traditionelle dreigliedrige System: In der Langzeitwirkung ließen sich keine differenziellen Wirkungen in Hinblick auf die Vorhersage erworbener Schulabschlüsse, des Berufsprestiges oder Einkommens in Abhängigkeit davon identifizieren, welches Schulsystem die Schülerinnen und Schüler durchlaufen hatten (Fend 2009a, b, 2014; Georg 2009). Inwieweit sich dieses Effektmuster allerdings auf die differenziellen Effekte von Zertifikat und Schulform innerhalb eines Systems übertragen lässt, wurde nicht geprüft.

Schuchart und Schimke (2019) untersuchten diese Frage in Hinblick auf die unterschiedlichen Zugangswege zum Abitur in querschnittlichen Analysen der Erwachsenenkohorte des Nationalen Bildungspanels (NEPS). Sie prüften, ob sich der direkte Weg zum Abitur über Gymnasien und Gesamtschulen und der indirekte, alternative Weg, z.B. über einen Realschulabschluss, im Hinblick auf das erreichte Berufsprestige unterschieden. Die Autoren fanden vor allem Unterschiede zwischen den verschiedenen Abschlussarten (Realschul-/Mittlerer Schulabschluss, Fachhochschulreife und Abitur) und kaum Unterschiede zwischen den beiden Wegen zum Abitur, und zwar auch nach Kontrolle von Kovariaten wie der Qualität der Abschlusszertifikate, Arbeitserfahrung und synchron erfasster kognitiver Leistungsvariablen. Wie die Autoren selbst konstatieren, bleibt offen, inwiefern kognitive und schulleistungsspezifische Unterschiede hinreichend bzw. angemessen kontrolliert wurden, da diese Variablen post-treatment (d.h. nach schulischer und beruflicher Ausbildung sowie Tätigkeit am Arbeitsmarkt) erhoben wurden. Dies ist insofern kritisch, da es Evidenz bezüglich reziproker Beziehungen zwischen kognitiven Funktionen und Berufszugehörigkeit gibt, und somit post-treatment erfasste Kompetenzen die Effekte verzerren könnten.

Die Effekte unterschiedliche Zugangswege zum (Fach-)Abitur auf das Einkommen hat Sterrenberg (2014) anhand des gleichen NEPS-Datensatzes untersucht. Ähnlich wie in den Analysen von Schuchart und Schimke (2019) für das Berufsprestige fanden sich vor allem Effekte der Zertifikate und nur schwache Hinweise auf Unterschiede zwischen den einzelnen Zugangswegen zum Abitur bzw. Fachabitur. Effekte schienen sich, wenn überhaupt, für diejenigen Personen anzudeuten, die vergleichsweise spät mit mehr als 25 Jahren ihr (Fach-)Abitur auf alternativen Wegen erworben hatten. Allerdings wurden in dieser Arbeit keinerlei kognitive oder motivationale Variablen einbezogen. Ebenso fehlt die explizite statistische Prüfung von Unterschieden zwischen den jeweiligen Zugangswegen. Dies lässt die spezifische Frage nach den Effekten der Schulformen der Sekundarstufe offen. Die Betrachtung der Unterschiede zwischen den Regressions-Koeffizienten legt allerdings nahe, 
dass auch ohne Kontrolle kognitiver Voraussetzungen allenfalls geringe Unterschiede zwischen den unterschiedlichen Zugangswegen zum Abitur nachzuweisen sein dürften.

\section{Die vorliegende Studie}

Vor dem Hintergrund der intensiven Diskussion um Schulformen und Schulreformen, die im Kern die Öffnung und Flexibilisierung der Zertifizierung des Sekundarschulwesen betreffen, erscheint es angezeigt, zu prüfen, inwiefern Schulformeffekte für zentrale Facetten des sozioökonomischen Erfolgs im Erwachsenenalter, d.h. das spätere erreichte berufliche Prestige, Einkommen und das Risiko der Arbeitslosigkeit, von Bedeutung sind. Hier setzt die vorliegende Studie an. Sie untersucht anhand längsschnittlicher Daten die Langzeitwirkungen unterschiedlicher Schulformen bis in das junge Erwachsenenalter (im Alter von knapp 31 Jahren) hinein. Die Studie geht damit auch der Frage nach, inwiefern die in Deutschland früh getroffenen Übergangsentscheidungen für die unterschiedlichen Schulformen der Sekundarstufe direkte Konsequenzen für den späteren beruflichen Erfolg haben.

Für die vorliegende Untersuchung wurde die Stichprobe der längschnittlichen large-scale Studie Bildungsverläufe und psychosoziale Entwicklung im Jugend-und jungen Erwachsenenalter (BIJU) genutzt. Die Studienteilnehmerinnen und -teilnehmer wurden nach dem Übergang in das Sekundarschulsystem in der 7. Klasse erstmals untersucht und zuletzt zu Beginn ihres vierten Lebensjahrzehnts im Alter von knapp 31 Jahren. Der Datensatz wurde genutzt, um insbesondere zu prüfen, inwiefern es zu unterschiedlichen Effekten der Schulformen einerseits und Abschlusszertifikaten andererseits für den späteren sozioökonomischen Erfolg kommt. Anders als in den bislang vorliegenden Arbeiten werden gleichzeitig alle vier großen Schulformen der Sekundarstufe 1 einbezogen, also Haupt-, Real-, Gesamtschulen und Gymnasien. Darüber hinaus wird weiterführend geprüft, inwiefern sich die Effektmuster multivariat verändern, also wenn arbeitsmarktbezogene Erfahrungen (beruflich-universitäre Ausbildung, Berufserfahrungen) bzw. die individuellen Ausgangsvoraussetzungen (sozialer und kognitiv-motivationaler Hintergrund) berücksichtigt werden. Jüngere Analysen von Becker et al. (2019) weisen darauf hin, dass Effekte dieser Variablen stark mit den spezifischen Dimensionen sozioökonomischen Erfolgs zusammenhängen, gleichzeitig aber ebenso stark mit Aspekten von Bildung konfundiert sind und somit berücksichtigt und geprüft werden sollte, welche geteilten bzw. unabhängigen Effekte sich hierbei finden lassen. In der vorliegenden Arbeit werden als Indikatoren des multidimensional zu verstehenden Konstrukts des sozioökonomischen Erfolges der berufliche Status, Einkommen und Arbeitslosigkeit im jungen Erwachsenenalter untersucht.

Ausgehend vor allem von humankapital- und filtertheoretischen Überlegungen und vorangehenden Befunden nehmen wir an, dass den Zertifikaten eine zentrale Stellung in der Prädiktion des beruflichen Erfolges zukommen sollte. Da das deutsche System neben der universitär-beruflichen Bildung auch eine breite berufliche Bildung in Form des dualen Systems und der schulischen beruflichen Bildung vorsieht und der Zugang zum Arbeitsmarkt verhältnismäßig stark über spezifische 
Qualifikationen reglementiert ist, gehen wir davon aus, dass berufliche Bildung von besonderer Bedeutung sein sollte, zumindest im Vergleich zu allgemeinbildenden Qualifikationen. Bezüglich der spezifischen Frage, ob sich darüber hinaus auch weiterführende Effekte für die Schulformen im Sekundarbereich finden, nehmen wir an, dass sich eher keine oder - wenn überhaupt - lediglich geringe Effekte nachweisen lassen. Zwar wurden gerade in Hinblick auf die Hauptschulen immer wieder ungünstige Effekte diskutiert, jedoch vermuten wir, dass dies vor allem auf die Konfundierung zwischen Schulform und Abschlusszertifikat zurückzuführen ist. Die jüngsten Analysen, die allerdings auf querschnittlichen Studien basieren, legen eine derartige Konfundierung zumindest in Hinblick auf die Hochschulzugangsberechtigungen nahe (Schuchart und Schimke 2019; Sterrenberg 2014). Aber auch auf der systemischen Ebene fielen Effekte von Strukturen longitudinal eher gering aus bzw. ließen sich nicht belegen (Fend 2014).

\section{Methode}

\subsection{Studiendesign und Stichprobe}

Die Stichprobe entstammt der BIJU-Studie, die federführend am Max-Planck-Institut für Bildungsforschung durchgeführt wurde. Die erste Erhebungswelle wurde 1991 zu Beginn der 7. Klassen durchgeführt (vgl. Tab. 1). In der vorliegenden Arbeit nutzen wir maßgeblich Angaben aus den ersten beiden Wellen (Anfang und Mitte der 7. Klasse), der 4. Welle (Ende 10. Klasse), sowie der Welle 7, die nach der Schulzeit erhoben wurde (letzte Welle im Jahr 2009/2010).

Die Stichprobe wurde als eine nach Bundesland und Schulform stratifizierte Zufallsstichprobe von 212 Schulen gezogen, wobei wiederum pro Schule je zwei ganze Schulklassen gezogen wurden. Die Studie wurde in 4 Bundesländern durchgeführt: Nordrhein-Westfalen, Mecklenburg-Vorpommern, Sachsen-Anhalt und Berlin. In Berlin wurde die Untersuchung allerdings erst mit der 2. Erhebungswelle begonnen. Mit der Welle 2 nahmen $N=8043$ Schülerinnen und Schüler teil, zu Welle 4 mit dem Ende der 10. Klasse waren es noch 5386 (vgl. Tab. 1), was sowohl auf Ausfälle auf der Individual- als auch der Schulebene zurückzuführen war. Aufgrund der Auflösung der Klassenverbünde in der gymnasialen Oberstufe wurde zum Zeitpunkt des Übergangs in die Oberstufe an Gymnasien, der Gesamt- und Sekundarschulen

Tab. 1 Überblick über die Studienteilnahme von 1991 bis 2009/2010

\begin{tabular}{lllll}
\hline Jahr & Welle & $\begin{array}{l}\text { Klassenstufe/ } \\
\text { Beruf }\end{array}$ & Stichprobe (total, $N)$ & $\begin{array}{l}\text { Teilnahme in Wel- } \\
\text { le } 7(N)\end{array}$ \\
\hline 1991 & 1 & 7 & 5944 & 1317 \\
1992 & 2 & 7 & 8043 & 1783 \\
1995 & 4 & 10 & 5386 & 2308 \\
1997 & 5 & $12 /$ Ausbild./Beruf & 8061 & 3615 \\
$2009 / 2010$ & 7 (kontakt.) $^{\mathrm{a}}$ & $/$ & 5292 & 4130 \\
\hline
\end{tabular}

12/Ausbild./Beruf in Schule (12. Klasse)/in beruflicher Ausbildung/auf dem Arbeitsmarkt

a 7 (kontakt.) Personen von denen eine Kontaktadresse verfügbar war 
ein Oversampling durchgeführt und alle Schülerinnen und Schüler der jeweiligen gymnasialen Oberstufe in die Stichprobe aufgenommen und getestet. Entsprechend wurde die Stichprobe um knapp 3000 Schülerinnen und Schüler vergrößert, so dass an der Welle 5 abermals $N=8061$ Schülerinnen und Schüler teilnahmen. Adressen wurden sowohl in der 10. als auch in der 12. Jahrgangstufe erhoben, wodurch zu Welle 7 noch $N=5292$ valide Adressen identifiziert werden konnten. Hiervon antworteten $N=4130$ Studienteilnehmerinnen und -teilnehmer. Alle Personen, die zu W7 kontaktierbar waren, bilden die Stichprobe, die den hier vorgestellten Analysen zugrunde liegen (weitere Details zur Stichprobe vgl. Becker et al. 2019).

In Tab. 2 wird der Datenausfall näher beschrieben. Entsprechend des längsschnittlichen Verlaufes ist der Datenausfall teilweise selektiv. Aber sofern direkte Vergleiche möglich sind, erscheint das Sample der BIJU-Studie in Hinblick auf die längsschnittliche Panelmortalität vergleichbar mit ähnlichen Studien (vgl. z. B. Damian et al. 2015; Fend et al. 2009; Spengler et al. 2015, 2018). Prinzipiell ist der Datenausfall in den früheren Wellen höher, maßgeblich durch Klassenwiederholungen, Beendigung der Schule nach der Pflichtschulzeit in der 9. Klasse, Schulwechsel und auch der Beendigung der Studienteilnahme ganzer Schulen bedingt. Entsprechend bilden die aus der Studie ausscheidenden Schülerinnen und Schüler eine negativ selektierte Stichprobe. Die Unterschiede liegen für diesen frühen Teilnehmer-Dropout zwischen $d=0,40$ für kognitive Grundfähigkeiten und $d=0,22$ hinsichtlich des elterlichen Hintergrundes (Prestige des väterlichen Berufes; vgl. Tab. 2). Entsprechend der kürzeren Studiendauer, der kognitiv und sozial günstigeren Zusammensetzung der Stichprobe sowie einer optimierten Adresserfassung ist der Ausfallprozess für die Stichprobe der 5. Welle (inklusive des Oversamplings der Schülerinnen und Schüler der gymnasialen Oberstufen) weniger selektiv. Zwar finden sich auch hier Hinweise auf eine positive Auswahl etwa in Hinblick auf kognitive Merkmale (z. B. günstigere Mathematikleistungen in der 12. Klasse mit $d=0,16$ ), aber in Hinblick auf soziale Hintergrundmerkmale lassen sich kaum Hinweise auf substantielle Un-

Tab. 2 Längsschnittliche Stichprobenselektivität: Wellenspezifische Vergleiche zwischen Schülerinnen und Schülern, die an der 7. Welle teilnahmen vs. nicht teilnahmen

\begin{tabular}{|c|c|c|c|c|c|c|c|c|c|c|c|c|c|c|c|}
\hline & & & Total & & & $\begin{array}{l}\text { Keine } \\
\text { W7 }\end{array}$ & eilnahn & & Teilna & ime W7 & & & & & \\
\hline Konstrukt & Jahr & Welle & $N$ & $M$ & $S D$ & $N$ & $M$ & $S D$ & $N$ & $M$ & $S D$ & $F$ & $(d f)$ & $p$ & $d$ \\
\hline \multicolumn{16}{|l|}{ Kognitiv } \\
\hline $\begin{array}{l}\text { Figurale } \\
\text { Intelli- } \\
\text { genz }\end{array}$ & 1991 & 1 & 5160 & 0,36 & 1,72 & 3955 & 0,20 & 1,72 & 1205 & 0,89 & 1,64 & 150,5 & $\begin{array}{l}(1 ; \\
5158)\end{array}$ & $<0,001$ & 0,40 \\
\hline $\begin{array}{l}\text { Figurale } \\
\text { Intelli- } \\
\text { genz }\end{array}$ & 1995 & 4 & 4842 & 2,05 & 1,38 & 2707 & 1,85 & 1,44 & 2135 & 2,30 & 1,26 & 124,5 & $\begin{array}{l}(1 ; \\
4840)\end{array}$ & $<0,001$ & 0,32 \\
\hline $\begin{array}{l}\text { Mathe- } \\
\text { matik }\end{array}$ & 1997 & 5 & 6522 & 2,23 & 0,91 & 3923 & 2,17 & 0,94 & 2599 & 2,32 & 0,87 & 40,2 & $\begin{array}{l}(1 ; \\
6520)\end{array}$ & $<0,001$ & 0,16 \\
\hline \multicolumn{16}{|c|}{ Elterlicher sozioökonomischer Hintergrund } \\
\hline $\begin{array}{l}\text { Treiman } \\
\text { Index }^{1}\end{array}$ & 1992 & 2 & 6559 & 44,60 & 12,96 & 4680 & 43,85 & 12,76 & 1522 & 46,66 & 13,21 & 54,8 & $\begin{array}{l}(1 ; \\
6200)\end{array}$ & $<0,001$ & 0,22 \\
\hline $\begin{array}{l}\text { Treiman } \\
\text { Index }\end{array}$ & 1997 & 5 & 5989 & 50,23 & 13,78 & 3131 & 50,21 & 13,65 & 2851 & 50,26 & 50,23 & 0,0 & $\begin{array}{l}(1 ; \\
5980)\end{array}$ & 0,885 & 0,00 \\
\hline
\end{tabular}


terschiede finden (z. B. $d=0,004$ für Prestige des väterlichen Berufes). Entsprechend der typischen Antwortmuster findet sich ein höherer Anteil von Frauen (60,2\%) in der Stichprobe. Teilnehmende Männer sind tendenziell positiv sozial selektiert (weitere demographische Angaben sowie weitere Angaben zu dropout; vgl. Bachsleitner et al. 2018; Radmann et al. 2017).

Die Testungen wurden bis zur 4. Welle (für Schülerinnen und Schüler der gymnasialen Oberstufe auch in der 5. Erhebungswelle) von geschulten Testleiterinnen und Testleitern im Klassenverband, alle nachfolgenden Befragungen postalisch durchgeführt. Die Tests und Schülerbefragungen wurden in allen schulischen Assessments an zwei aufeinander folgenden Tagen administriert. Die Studie wurde von den jeweiligen Bildungsministerien und den Ethikkommissionen der beteiligten Forschungseinrichtungen bewilligt.

\subsection{Instrumente}

\subsubsection{Abhängige Variable: Sozioökonomischer Erfolg}

Berufsprestige In Welle 7 wurden die Teilnehmerinnen und Teilnehmer nach ihrem zuletzt ausgeübten Beruf gefragt. Diese Angaben wurden nach der International Standard Classification of Occupations (ISCO 2008; International Labor Office 2012) kodiert. Die kodierten Daten wurden wiederum genutzt, um den sozioökonomischen Status gemäß des International Socio-Economic Index of Occupational Status (ISEI; Ganzeboom 2010) zu kodieren. Die ISCO-08-Kodierungen und zugehörige Transformationen in ISEI-Werte wurden durch das Data Processing Center (DPC) in Hamburg vorgenommen. Der mittlere sozioökonomische Status der Stichprobe betrug $M=51,9$ ( $S D=19,6$; gewichtete Stichprobe).

Einkommen Zudem wurden die Teilnehmerinnen und Teilnehmer zu ihrem gegenwärtigen Einkommen (brutto, in Euro) zu Welle 7 befragt. Dieses wurde, wie in Analysen zu Einkommen gängig, logarithmisch transformiert, da dies die Verteilungsform normalisiert und die Analysen weniger sensitiv gegenüber einzelnen Ausreißerwerten macht (vgl. z. B. Strenze 2007). Personen ohne Einkommen wurden aus den Analysen ausgeschlossen. Das mittlere Einkommen der Stichprobe betrug $M=2556 €(S D=2401 €$; Einkommen, logarithmiert $M=7,66, S D=0,62$; gewichtete Stichprobe).

Arbeitslosigkeit Zudem wurden die Teilnehmerinnen und Teilnehmer zu ihrem gegenwärtigen Erwerbsstatus zu Welle 7 befragt. Als arbeitslos wurden diejenigen Personen klassifiziert, die sich als arbeitslos bezeichneten und entweder Arbeitslosengeld I und II/Grundsicherung oder keine staatliche Unterstützungsleistung bezogen. Insgesamt traf dies auf $N=243$ Personen (gewichtete Stichprobe) zu.

\subsubsection{Prädiktoren. Schulabschlüsse, Schulformen, Berufserfahrung}

Bildung In Welle 7 wurden der final erreichte schulische und berufliche (universitäre) Abschluss der Teilnehmerinnen und Teilnehmer erfasst. Hierbei wurden für die 
allgemeinbildenden Schulabschlüsse Hauptschul-, Mittlerer (Real-)Schulabschluss, Fachhochschulreife und Abitur unterschieden und als Dummy-Variablen verwendet (Referenzkategorie: Mittlerer Schulabschluss). Berufliche Bildung wurde fünfstufig erfasst: Es wurde unterschieden, ob keine berufliche Qualifikation vorlag oder ob eine Lehre, ein Meister-/Techniker-/gleichwertiger Abschluss, ein Studium oder eine Promotion als letzte berufliche Ausbildung absolviert wurde (als Dummy-Variablen; Referenzkategorie: Lehre). Als weitere Indikatoren für Bildung und Bildungserfolg wurden Noten in die Vorhersagen aufgenommen, die als Indikatoren für die Qualität des jeweils erworbenen Abschlusses dienen. Dies erfolgte wiederum getrennt in zwei Facetten, d.h. es wurde die Note für den höchsten allgemeinbildenden Schulabschluss und zudem die Note des letzten beruflichen bzw. universitären Abschlusses verwendet. Die Schulform selbst wurde danach erfasst, welche Schulform die Schülerinnen und Schüler in der 7. Klasse besuchten. Hauptschule, Realschule und Gymnasium gingen als Dummy-Variablen in die Analysen ein; die Gruppe der Gesamtschulen (integrierte Gesamtschulen, Sekundarschulen, Schulen mit mehreren Bildungsgängen) dienten als Referenzgruppe.

\subsubsection{Individueller und familiärer Hintergrund}

Kognitive Grundfähigkeiten Um die allgemeinen kognitiven Fähigkeiten in der späten Kindheit (zur 7. Klasse; also zu Beginn der schulischen Gliederung in der Sekundarstufe) zu kontrollieren, wurden vier Indikatoren zur latenten Modellierung herangezogen: aus dem KFT 4-13+ (Heller et al. 1985) wurden verbale und figurale Figurenanalogien-Subskalen verwendet (Welle 1). Aus Amthauers (1955) Intelligenz Strukturtest (IST) wurden die Subskalen zu numerischen und räumlichen Fähigkeiten eingesetzt (Welle 2). Beide Tests und vor allem die vier Subskalen sind Vorläuferversionen auch heute noch eingesetzter Intelligenztests (Amthauer et al. 2001; Heller und Perleth 2000; Liepmann et al. 2007). Die Reliabilitäten fielen von sehr gut bis lediglich befriedigend aus (figural: Cronbach's $\alpha=0,91$; verbal: Cronbach's $\alpha=0,82$; räumlich: Cronbach's $\alpha=0,68 / 0,74$ (Version A and B); numerisch: Cronbach's $\alpha=0,90$; jeweils mit Kuder-Richardson Formula 20), was jedoch für Intelligenztest-Subskalen nicht ungewöhnlich ist. Wie eingangs erwähnt wurden die vier Indikatoren für einen latenten, messfehlerbereinigten Faktor verwendet (vgl. Abschn. 4.3).

Schulleistung Zur Messung der akademischen Leistung in der 10. Klasse wurden drei standardisierte Leistungstests für die Domänen Mathematik, Englisch und Physik herangezogen. Die Testitems entstammen unterschiedlichen Studien: Im Wesentlichen wurden die Items den damaligen großen IEA-Studien entnommen (Baumert et al. 1997; Edelstein 1970; Husén 1967; Travers und Westbury 1989; Walker 1976) oder Items in Eigenentwicklung. Um den unterschiedlichen curricularen Anforderungen gerecht zu werden, wurden die Tests in allen drei Domänen jeweils taylored für Schulformen administriert (Versionen für Gymnasium/Realschule vs. andere: Englisch und Mathematik; Version für Gymnasium vs. andere: Physik). Die Reliabilitäten der Tests waren zufriedenstellend bis sehr gut mit Cronbachs $\alpha=0,76-78$ (Mathematik), $\alpha=0,89$ (Englisch) und $\alpha=0,76-77$ (Physik). Weiterführende Details 
zu den Tests finden sich bei Becker et al. (2012) sowie Tetzner (2014). Analog zu den kognitiven Grundfähigkeiten wurde ein latenter, messfehlerbereinigter Faktor der Schulleistungen verwendet (vgl. Abschn. 4.3).

Familiärer Hintergrund Der elterliche sozioökonomische Hintergrund wurde einerseits über den beruflichen Status, andererseits über die Bildung der Eltern erfasst. Analog zur oben beschriebenen Kodierung der Berufe der Zielpersonen wurden die Angaben zum zuletzt ausgeübten Beruf der Eltern einbezogen. Der Beruf der Mutter und des Vaters wurden getrennt verwendet. Ebenso wurden die Angaben der frühen Wellen (Wellen 1-3) sowie die Angaben zu Welle 5 getrennt, um eine bessere Kontrolle von Messfehlern zu ermöglichen. Die Angaben zu den Berufen der Eltern der frühen Wellen 1-3 wurden nach der International Standard Classification of Occupations (ISCO-68; International Labor Office 1968) kodiert, die späteren Angaben aus Welle 5 nach ISCO-88 (ISCO-88; International Labour Organization 1990). Diese ISCO-Codes wurden jeweils nach dem damals aktuellen Stand zum Prestige des Berufes nach Treiman (1977) bzw. in der späteren Fassung von Ganzeboom und Kollegen für den ISCO-88 (Ganzeboom und Treiman 2003; Geis 2011) metrisiert. Die Kodierungen wurden durch das Zentrum für Umfragen, Methoden und Analysen (ZUMA) in Mannheim vorgenommen und nach dem damaligen Stand der Wissenschaft umgesetzt, was z. B. detailliertere GESIS-Zusatzcodes für die Berufskodierungen in Deutschland umfasste (z. B. Zusatzkodierungen für Berufe in der DDR; vgl. Geis 2011). Die Angaben stammten jeweils aus den Berichten der Schülerinnen und Schüler über ihre Eltern. Zudem wurde die elterliche Bildung einbezogen und über zwei Indikatoren erfasst: einerseits als Anzahl der Hochschulzugangsberechtigungen, andererseits als Anzahl universitärer Qualifikationen der Eltern $(0=$ kein Abitur/ Universitätsabschluss bis $2=$ beide Eltern Abitur/Universitätsabschluss). Analog zu den kognitiven Maßen wurden diese 6 Indikatoren herangezogen, um einen latenten, messfehlerbereinigten Faktor für den elterlichen Hintergrund zu schätzen. Zusätzlich wurden migrationsbezogene Charakteristika der Familie mittels des Geburtslandes der Eltern modelliert. Als Proxis für die Kodierung eines Migrationshintergrundes unterschieden wir, inwiefern ein Elternteil $(N=204)$ oder beide Elternteile im Ausland geboren wurden $(N=169$; Referenzkategorie dieser beiden Dummy-Variablen: kein Elternteil im Ausland geboren). Aufgrund der Fallzahlen wurde die letztgenannte Kategorie nicht weiter differenziert.

Berufliche Aspirationen Als ein Indikator für die motivationalen Unterschiede wurden die beruflichen Aspirationen als weiterer Prädiktor mit einbezogen. Hierfür verwendeten wir die Angaben des beruflichen Erstwunsches am Ende der 7. Klasse, die wiederum offen erfasst wurden und über eine Kodierung als ISCO-Codes (ISCO68; International Labor Office 1968) in metrisch skalierte Angaben zum Prestige des Berufes nach Treiman (1977) skaliert wurden.

Gender Das Geschlecht der Schülerinnen und Schüler wurde über die Wellen hinweg gepoolt und ging in Form eines Dummys $(1=$ weiblich, $0=$ männlich $)$ in die Analysen ein (zu weiterführenden geschlechtsspezifischen Analysen siehe zudem Becker et al. 2019). 
Kontrollvariablen In allen Analysen wurden die Bundesländer als Kontrollvariablen mitgeführt, um für regionale Unterschiede, vor allem in der Schulstruktur, Einkommen und Beschäftigungsstruktur, zu kontrollieren.

\subsection{Statistische Analysen}

In einem ersten Schritt wurde die latente Modellierung der Prädiktoren der kognitiven Grundfähigkeiten, akademischen Leistung und des elterlichen sozioökonomischen Hintergrunds der Kinder vorgenommen, die jeweils mit 4, 3 und 6 Indikatoren gemessen wurden. Zur Beurteilung der Modellpassung haben wir absolute Fit-Kriterien herangezogen, die unabhängig von der Stichprobengröße zu interpretieren sind, d.h. RMSEA, CFI und SRMR, und uns hierbei an den gemeinhin akzeptierten cut off-Kriterien nach Hu und Bentler (1999) und Schermelleh-Engel et al. (2003) orientiert (RMSEA $<0,05-0,08$; CFI $>0,90-0,95$; SRMR $<0,05-0,10$ ). Das Modell für Intelligenz erzielte hierbei einen Fit von RMSEA=0,023; CFI=0,997; $\mathrm{SRMR}=0,012$ und das des familiären Hintergrundes RMSEA $=0,050 ; \mathrm{CFI}=0,921$; SRMR $=0,067$ (Schulleistung ist saturiert). Das gemeinsame Modell aller drei latenter Faktoren erzielte ebenso einen akzeptablen Modellfit mit RMSEA $=0,026$; $\mathrm{CFI}=0,942$ und $\mathrm{SRMR}=0,048$.

Neben den deskriptiven Analysen, die Auskunft über die Zusammenhänge zwischen besuchter Schulform in der Sekundarstufe und späteren allgemeinbildenden und beruflichen Abschlusszertifikaten geben, wurde in einem weiteren Schritt unter Einbezug der latenten und weiterer manifester Variablen multivariate Regressionsanalysen durchgeführt, um die spezifischen bzw. multivariaten Effekte (d.h. nach Kontrolle der jeweils anderen Variablen) für die Vorhersage des beruflichen Status, Einkommens und der Arbeitslosigkeit im jungen Erwachsenenalter zu schätzen. Die Analysen wurden hierarchisch aufgebaut, so dass die Effekte der Schulstrukturen und der Zertifikate sowohl unabhängig und gemeinsam, als auch unter Kontrolle der Leistungs- und soziographischen Hintergrundvariablen erfasst sind. In allen Regressionsanalysen wurde zudem das Bundesland kontrolliert, um regionalen Unterschieden Rechnung zu tragen. Für die Analyse der Arbeitslosigkeit zogen wir aufgrund der größeren Robustheit und der besseren Interpretierbarkeit in multivariaten Analysemodellen das robustere lineare Wahrscheinlichkeitsmodell heran (zur Problematik von logistischen Regressionen in multivariaten Analysen, vgl. u.a. Baumert et al. 2018; Mood 2010).

Alle Analysen wurden mit Mplus 7.4 (Muthén und Muthén 2013) durchgeführt. Standardfehler wurden mittels der Analyseoption type $=$ complex in Mplus umgesetzt, in dem die stratifizierte Struktur der Daten berïcksichtigt werden kann. Dies erlaubte auch Stichprobengewichte einzubeziehen, mit denen die unterschiedlichen Ziehungswahrscheinlichkeiten je nach Schulform und Bundesland berücksichtigt werden konnten.

Fehlende Daten, die, wie oben erläutert, zumindest in Teilen nicht zufällig fehlen, wurden durch multiple Datenimputationen berücksichtigt (Graham 2009; Little und Rubin 2002), die weniger restriktive Annahmen als z. B. Ausschlussverfahren bezüglich des Datenausfalls zu machen (Lüdtke und Robitzsch 2011). Für die Imputationen wurden alle Kontroll- und Analysevariablen einbezogen (für latente Faktoren 
auf Ebene der Indikatoren). Die Mehrebenenstruktur wurde mithilfe der Schulmittelwerte berücksichtigt. Es wurden $m=100$ Imputationen im Programm R (R Core Team 2019) mit den Routinen mice (van Buuren und Groothuis-Oudshoorn 2011) und mitml (Grund et al. 2016) vorgenommen. Die imputierten Datenanalysen wurden in Mplus mit der Analyseoption type = imputation einbezogen, die es ermöglicht, auch die between imputation-Varianz für die Schätzung der Standardfehler nach den Regeln von Rubin (Rubin 1987) zu berücksichtigen. Wir haben zudem überprüft, inwiefern die Ergebnisse zwischen unserem Imputations-basierten Verfahren im Vergleich zum full information maximum likelihood (FIML)-Ansatz ähnlich ausfielen, was der Fall war. Es ergaben sich nahezu identische Ergebnisse (zur weiterführenden Sensitivitätsprüfung bezüglich unterschiedlicher Umsetzungen; vgl. Abschn. 6.1).

\section{Ergebnisse}

\subsection{Deskriptive Betrachtung}

Ganz grundlegend geben die Tab. 3 und 4 die bivariaten Zusammenhänge zwischen der besuchten Schulform und den jeweiligen erreichten schulischen und beruflichen Abschlüssen an (Tab. 3: nach allgemeinbildend; Tab. 4: beruflich). In beiden Tabellen ist zu erkennen, dass der Zusammenhang zwar mit einem Kontingenzkoeffizient von $C=0,64 ; p<0,001\left(\chi^{2}=2720,3 ; d f=12 ; p<0,001\right)$ eng, aber keineswegs deterministisch ausfiel. Zwar wurden zum Beispiel die meisten Hauptschulabschlüsse weiterhin an Hauptschulen erworben $(73,3 \%)$, jedoch qualifizierte sich lediglich der geringere Teil der Hauptschülerinnen und Hauptschüler mit einem Hauptschulabschluss $(N=121 ; 29,9 \%)$. Die meisten der $N=405$ Hauptschülerinnen und Hauptschüler erreichten im Laufe ihrer schulisch-beruflichen Karriere noch einen mittleren Schulabschluss $(N=188 ; 46,4 \%)$, ein kleinerer Teil auch das (Fach-)Abitur $(N=96 ; 23,7 \%)$. Umgekehrt wurden die meisten der Hochschulzugangsberechtigungen von Gymnasiastinnen und Gymnasiasten erworben (76,2\%); aber knapp ein Viertel der Hochschulzugangsberechtigungen wurde von Schülerinnen und Schülern

Tab. 3 Besuchte Schulform und erreichter allgemeinbildender Schulabschluss ( $N$; Spaltenprozent; gewichtete Rohdaten)

\begin{tabular}{|c|c|c|c|c|c|c|c|c|c|c|c|c|}
\hline \multirow[b]{2}{*}{ Schulform } & \multicolumn{2}{|c|}{$\begin{array}{l}\text { Kein } \\
\text { Abschluss }\end{array}$} & \multicolumn{2}{|c|}{$\begin{array}{l}\text { Volks-/Haupt- } \\
\text { schulabschluss }\end{array}$} & \multicolumn{2}{|c|}{$\begin{array}{l}\text { Mittlerer } \\
\text { Schulabschluss }^{\mathrm{a}}\end{array}$} & \multicolumn{2}{|c|}{$\begin{array}{l}\text { Fachhochschul- } \\
\text { reife }\end{array}$} & \multicolumn{2}{|l|}{ Abitur } & \multicolumn{2}{|c|}{ Gesamt } \\
\hline & $N$ & $\%$ Spalte & $N$ & $\%$ Spalte & $N$ & $\%$ Spalte & $N$ & $\%$ Spalte & $N$ & $\%$ Spalte & $N$ & $\%$ Spalte \\
\hline $\begin{array}{l}\text { Haupt- } \\
\text { schule }\end{array}$ & 0 & 0 & 121 & 73,3 & 188 & 12,2 & 85 & 14,1 & 11 & 0,7 & 405 & 10,3 \\
\hline Realschule & 0 & 0 & 0 & 0,0 & 564 & 36,6 & 261 & 43,4 & 146 & 8,9 & 971 & 24,6 \\
\hline $\begin{array}{l}\text { Gesamt- } \\
\text { schule }^{b}\end{array}$ & 1 & 100 & 44 & 26,7 & 706 & 45,8 & 160 & 26,6 & 234 & 14,3 & 1145 & 29,0 \\
\hline $\begin{array}{l}\text { Gymna- } \\
\text { sium }\end{array}$ & 0 & 0 & 0 & 0,0 & 82 & 5,3 & 96 & 15,9 & 1250 & 76,2 & 1428 & 36,2 \\
\hline Gesamt & 1 & 100 & 165 & 100 & 1540 & 100 & 602 & 100 & 1641 & 100 & 3949 & 100 \\
\hline
\end{tabular}

${ }^{a}$ mittlerer Schulabschluss: mittlerer Schulabschluss/mittlere Reife/Realschulabschluss

${ }^{\mathrm{b}}$ Gesamtschule: Gesamt-/Sekundarschule/Schule mit mehreren Bildungsgängen 
Tab. 4 Besuchte Schulform und erreichter beruflicher Abschluss ( $N$; Spaltenprozent; gewichtete Rohdaten)

\begin{tabular}{|c|c|c|c|c|c|c|c|c|c|c|c|c|}
\hline & \multicolumn{2}{|c|}{$\begin{array}{l}\text { Keine berufli- } \\
\text { che Qualifikati- } \\
\text { on }\end{array}$} & \multicolumn{2}{|l|}{ Lehre } & \multicolumn{2}{|c|}{$\begin{array}{l}\text { Meister/ } \\
\text { Techniker/ } \\
\text { gleichwertiger } \\
\text { Fachabschluss }\end{array}$} & \multicolumn{2}{|c|}{ Studium } & \multicolumn{2}{|c|}{ Promotion } & \multicolumn{2}{|c|}{ Gesamt } \\
\hline & $N$ & $\%$ & $N$ & $\%$ & $N$ & $\%$ & $N$ & $\%$ & $N$ & $\%$ & $N$ & $\%$ \\
\hline $\begin{array}{l}\text { Haupt- } \\
\text { schule }\end{array}$ & 49 & 29,9 & 252 & 12,4 & 58 & 16,2 & 29 & 2,5 & 2 & 1,9 & 390 & 0,0 \\
\hline Realschule & 22 & 13,4 & 632 & 31,0 & 87 & 24,3 & 195 & 16,7 & 9 & 8,3 & 945 & 4,2 \\
\hline $\begin{array}{l}\text { Gesamt- } \\
\text { schule }^{\mathrm{a}}\end{array}$ & 37 & 22,6 & 780 & 38,3 & 134 & 37,4 & 158 & 13,5 & 1 & 0,9 & 1110 & 39,0 \\
\hline Gymnasium & 56 & 34,1 & 375 & 18,4 & 79 & 22,1 & 788 & 67,4 & 96 & 88,9 & 1394 & 15,2 \\
\hline Gesamt & 164 & 100 & 2039 & 100 & 358 & 100 & 1170 & 100 & 108 & 100 & 3839 & 100 \\
\hline
\end{tabular}

${ }^{\mathrm{a}}$ Gesamtschule: Gesamt-/Sekundarschule/Schule mit mehreren Bildungsgängen

erworben, die in der Mittelstufe kein Gymnasium besuchten. Für die Gymnasiastinnen und Gymnasiasten selbst impliziert dies, dass die Mehrheit mit mehr als $94 \%$ mindestens eine Fachhochschulreife erzielte. Die Real- und Gesamtschulen liegen erwartungsgemäß in der Mitte zwischen diesen beiden Extremen.

Ein ähnliches Bild zeichnete sich auch für die beruflichen Abschlüsse ab (vgl. Tab. 4), wenngleich hier der Zusammenhang zwischen besuchter Schulform und erworbenem Abschluss mit einem Kontingenzkoeffizienten von $C=0,46\left(\chi^{2}=1035,7\right.$; $d f=12 ; p<0,001)$ noch weniger systematisch ausfiel. Im Wesentlichen findet sich in allen Kategorien der beruflichen Abschlüsse jede besuchte Schulform vertreten, wenngleich die zu erwartenden systematischen Häufungen zu finden sind, d. h. Hauptschülerinnen und -schüler qualifizierten sich vor allem in der beruflichen Lehre, Gymnasiastinnen und Gymnasiasten vor allem durch ein Studium. Schülerinnen und Schüler der anderen Schulformen liegen wiederum zwischen diesen beiden Extremen.

Tab. 5 gibt darüber hinaus die bivariaten, korrelativen Zusammenhänge zwischen den Schulformen, Schulabschlüssen und Noten, den individuellen Hintergrundvariablen und den sozioökonomischen Outcomes wieder. Man kann einerseits erkennen, dass die sozioökonomischen Outcomes des beruflichen Status, Einkommens und der Arbeitslosigkeit im jungen Erwachsenenalter mit den Schulformen als auch mit den schulischen Abschlüssen und Noten zusammenhingen, und ebenso mit den weiteren kognitiven, motivationalen und sozialen Hintergrundmerkmalen. Erwartungskonform wiesen fast alle Variablen statistisch signifikante Zusammenhänge untereinander auf, was entsprechend empirisch eine systematische multivariate Betrachtungsweise indiziert.

\subsection{Multivariate Analyseergebnisse}

Die multivariaten, hierarchisch aufgebauten Regressionsanalysen finden sich in den Tab. 6 für die Vorhersage des beruflichen Status, in Tab. 7 für die Vorhersage des Einkommens und in Tab. 8 für die Vorhersage der Arbeitslosigkeit im jungen Erwachsenenalter. Betrachtet man zunächst die Vorhersage des beruflichen Status (Tab. 6), 


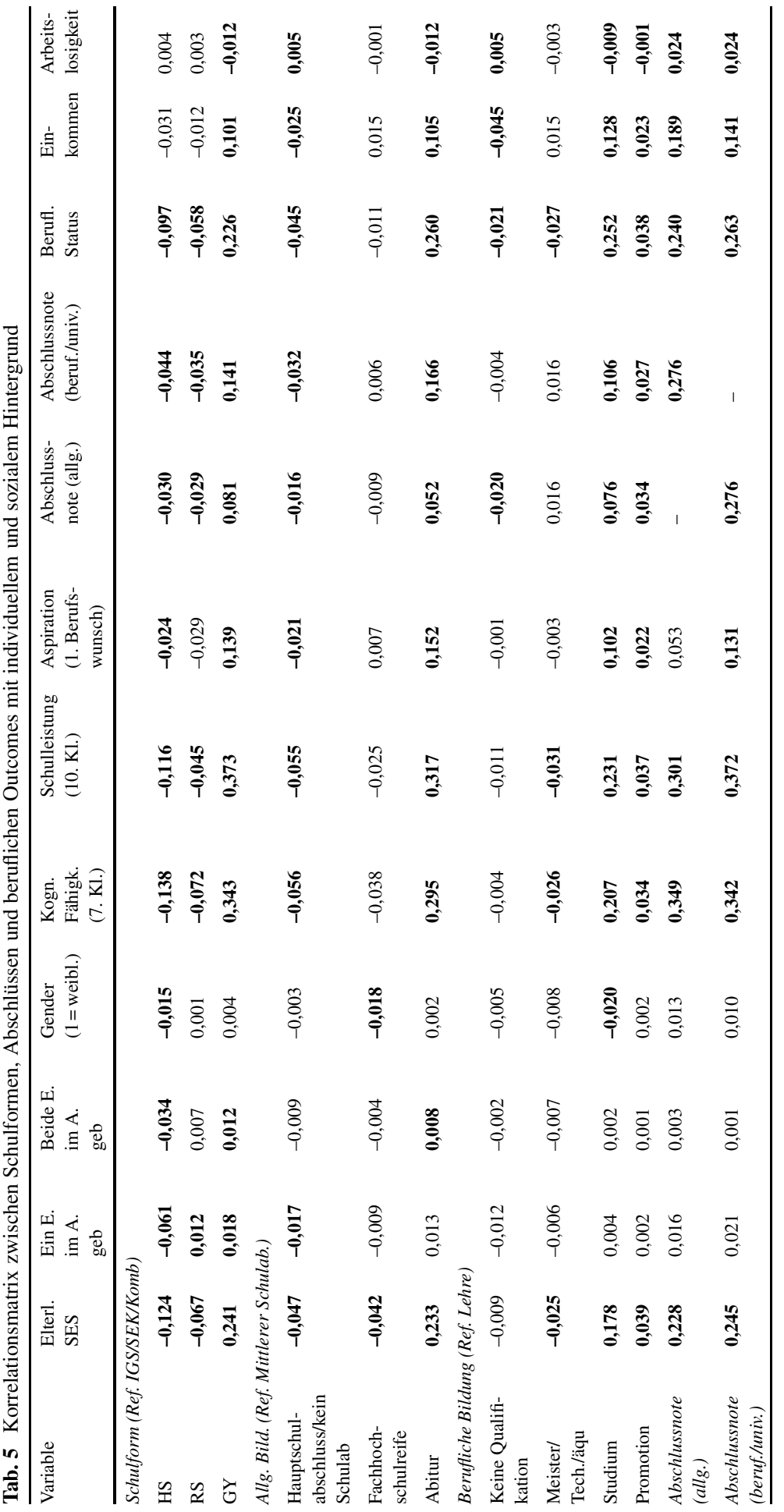




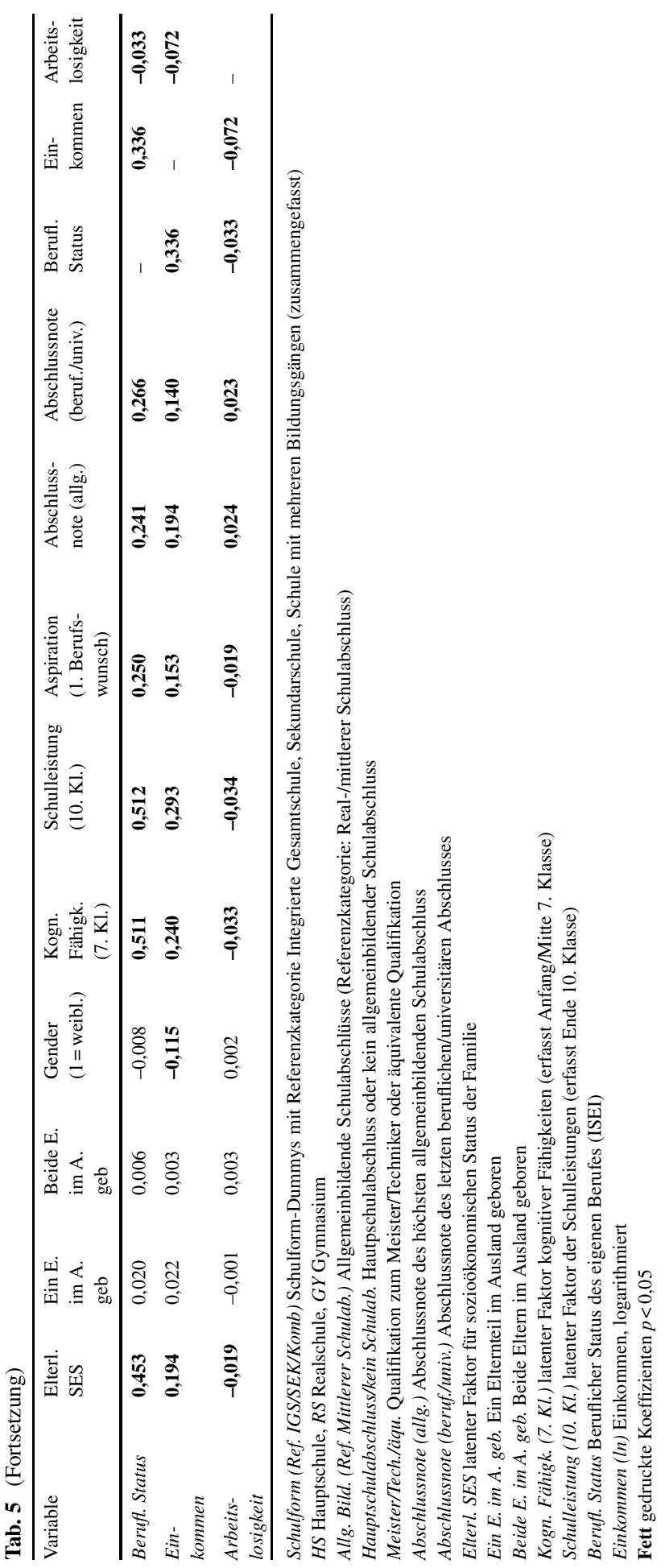


so zeigte sich ein negativer Effekt des Besuchs einer Hauptschule und ein positiver Effekt des Besuchs eines Gymnasiums, jeweils im Vergleich zur Referenzgruppe der Gesamt- und Sekundarschulen und Schulen mit mehreren Bildungsgängen (Modell M1). Realschulen unterschieden sich nicht signifikant von dieser Referenzgruppe. In der Vorhersage des beruflichen Status durch die allgemeinbildenden schulischen und beruflichen bzw. universitären Abschlüsse (Modell M2) fanden sich sowohl für den Hauptschulabschluss als auch für die Fachhochschulreife und das allgemeinbildende Abitur statistisch bedeutsame Effekte im Unterschied zum mittleren Schulabschluss. Hinsichtlich der unterschiedlichen beruflichen Ausbildungen zeigten sich im Vergleich zur Lehre keine statistisch bedeutsamen Unterschiede für Personen ohne berufliche Qualifikation oder mit Meister-/Techniker-/gleichwertigem Fachabschluss; wohl aber wiesen ein Studium bzw. eine Promotion einen jeweils statistisch bedeutsamen positiven Effekt auf.

In den weiterführenden multivariaten Analysen, in denen sowohl die Dummys für die besuchten Schulformen als auch gleichzeitig die tatsächlichen Qualifikationen in das Modell M3 eingeführt wurden, verringerten sich die Effekte deutlich: der des Besuchs einer Hauptschule verringerte sich um knapp die Hälfte, der Effekt des Gymnasiums um mehr als drei Viertel. Beide Effekte blieben aber statistisch signifikant. Durch zusätzliche Kontrolle der Abschlussnoten (Modell M4), in der die Note des höchsten allgemeinbildenden Schulabschlusses statistisch bedeutsam war, zeigten sich die Schulformeffekte kaum verändert. In einem weiteren Schritt, in dem zusätzlich auch die kognitiven und soziographischen Merkmale zur Prädiktion hinzugezogen wurden (M5), blieb lediglich ein Unterschied der Hauptschule bestehen. Für den beruflichen Status wurden im finalen Modell zudem der allgemeinbildende Schulabschluss ([Fach-]Abitur bzw. als Abschlussnote) sowie die universitären Abschlüsse (im Vergleich zur Lehre) sowie der sozioökonomische Hintergrund des Elternhauses statistisch signifikant. Die weiteren Variablen bezüglich des Migrationshintergrundes, des Geschlechts, der kognitiven und schulischen Leistungen sowie die beruflichen Aspirationen wurden in diesem multivariaten Modell nicht mehr statistisch signifikant.

Prima facie stellte sich das Effektmuster hinsichtlich der Vorhersage des Einkommens ähnlich dar (Tab. 7), wenngleich sich einige zentrale Unterschiede identifizieren lassen. In den Modellen M1 und M2 fanden sich statistisch bedeutsame Effekte sowohl für den Besuch eines Gymnasiums (M1) als auch für das Abitur und alle beruflichen Abschlüsse (M2). In Hinblick auf die Frage nach Schulstruktur vs. Qualifikation treten Unterschiede vor allem ab Modell M3 auf: Bei einer gleichzeitigen Prädiktion verliert sich der Effekt des Abiturs; statistische Signifikanzen lassen sich weiterhin für den Besuch eines Gymnasiums und die beruflichen Zertifikate finden. Wenn die Qualität der Zertifikate in Form von Noten (M4), sowie kognitive und soziale Hintergrundmerkmale (M5) zusätzlich in die Modelle aufgenommen wurden, verliert sich die statistische Bedeutsamkeit des Gymnasiums. Es bleiben lediglich die unterschiedlichen beruflichen Abschlüsse sowie die Noten der allgemeinbildenden Schulabschlüsse die statistisch bedeutsamen Prädiktoren. Von den Hintergrundmerkmalen war nur ein Faktor statistisch signifikant: für Frauen fand sich ein negativer Effekt im Vergleich zu den Männern. 


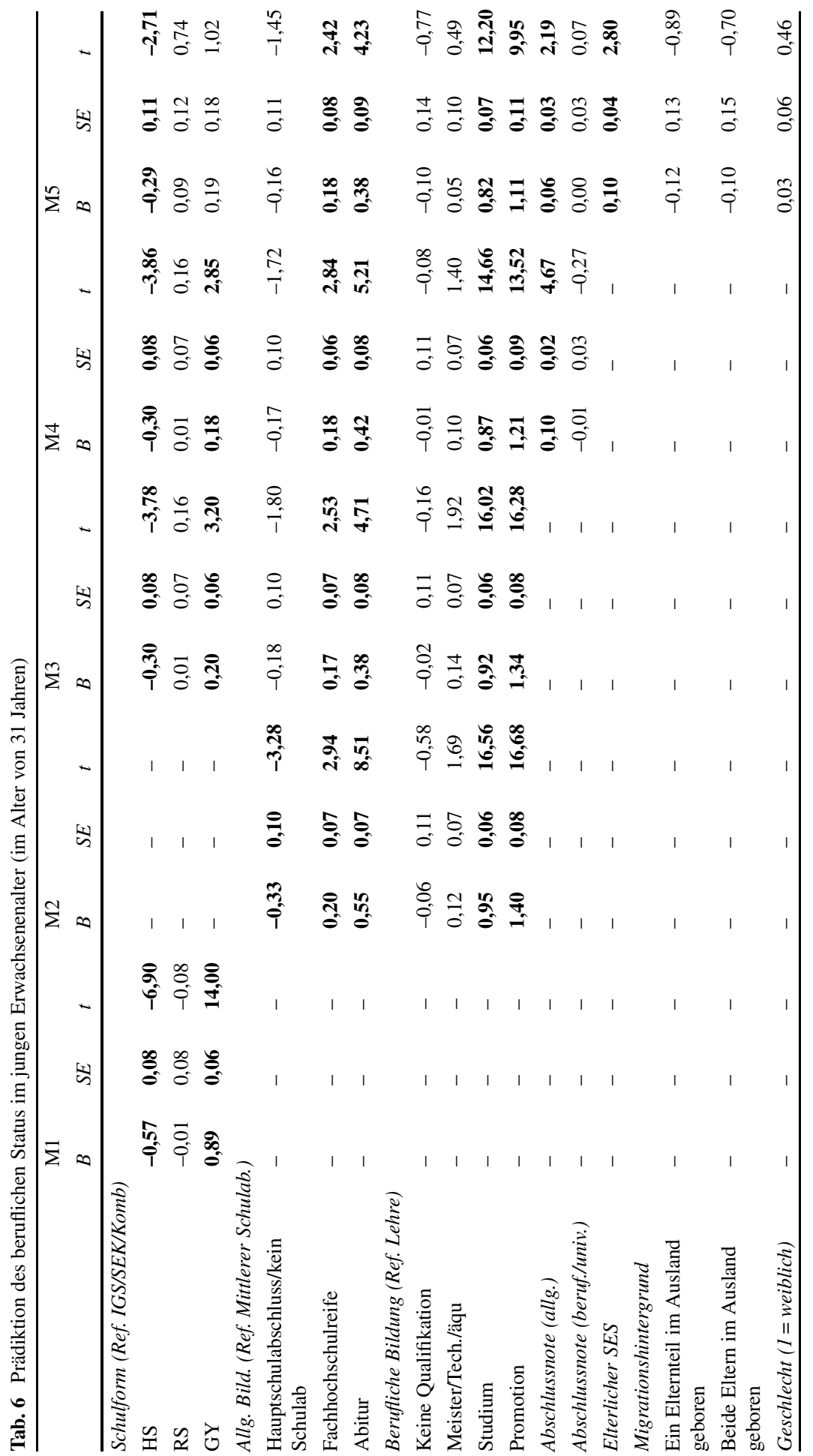




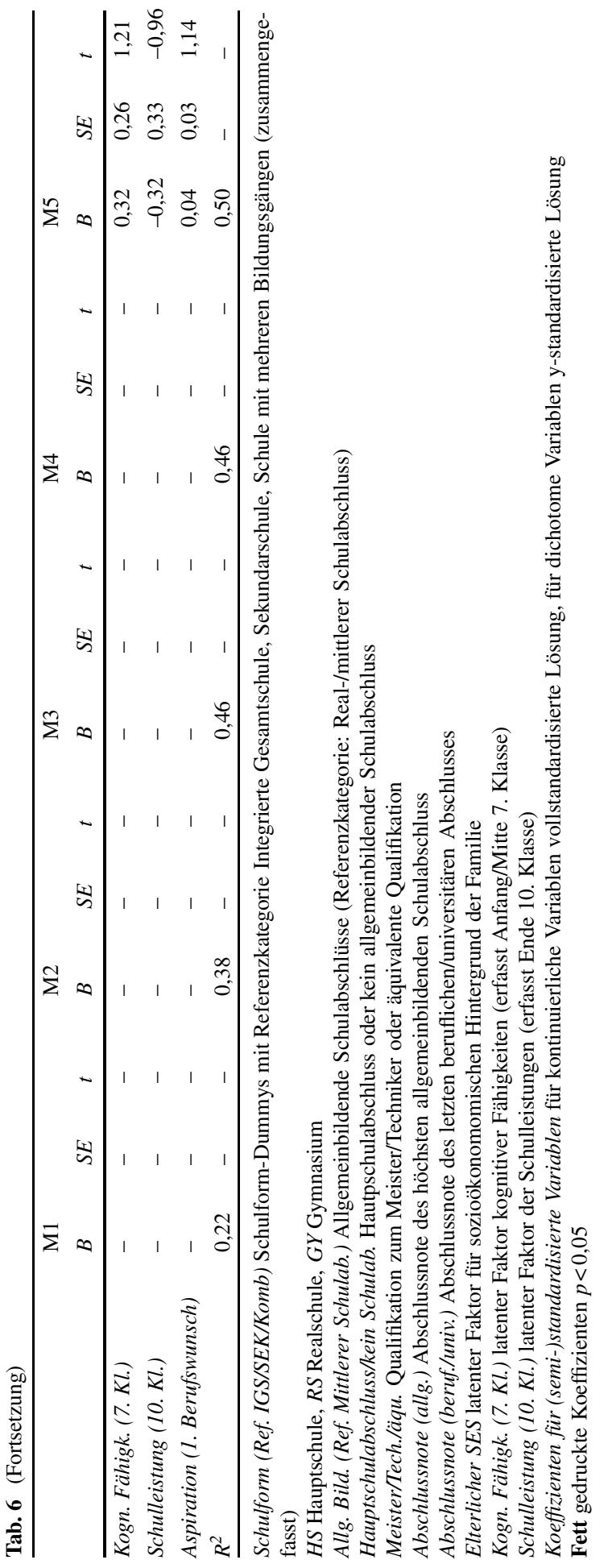




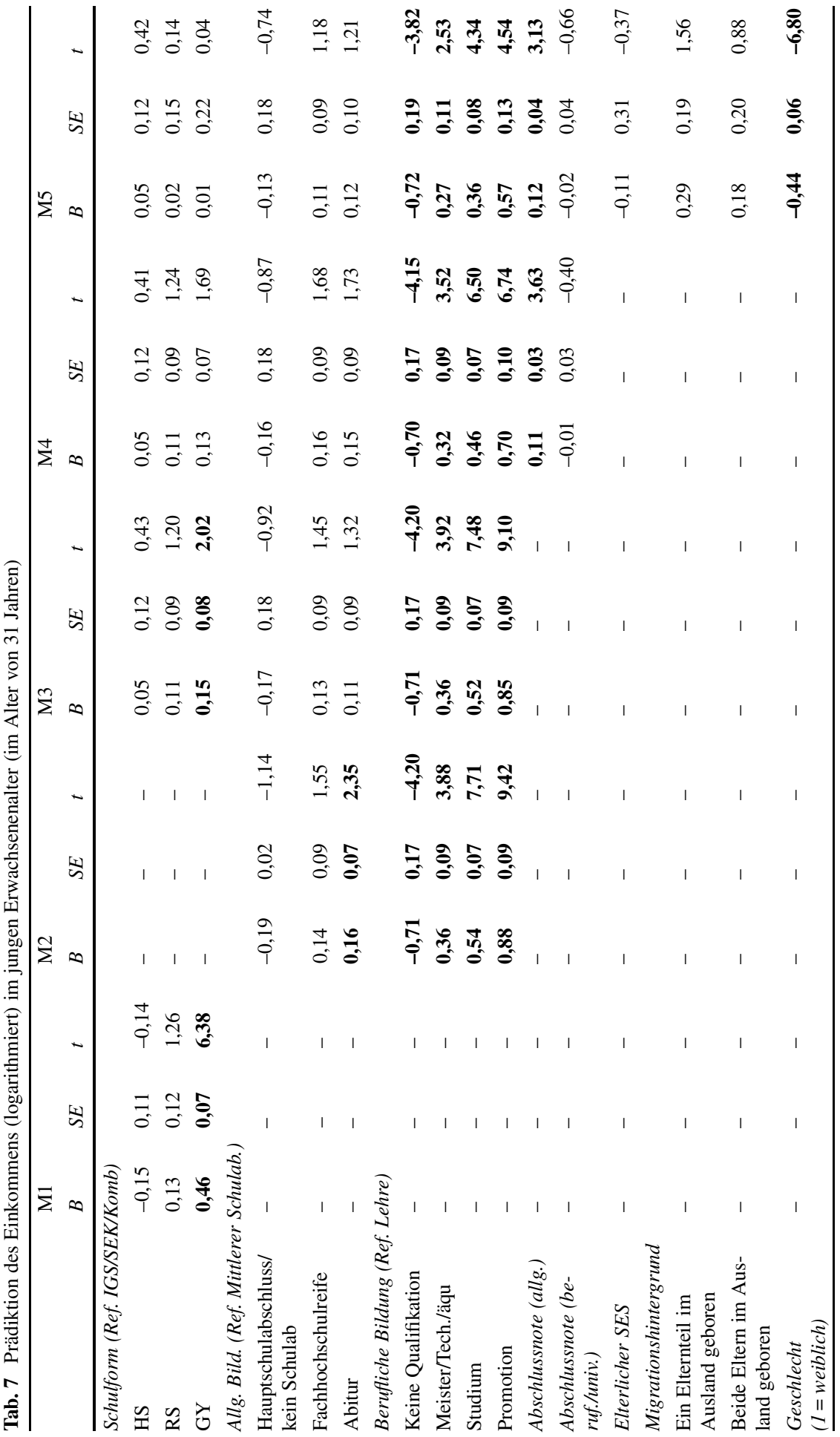




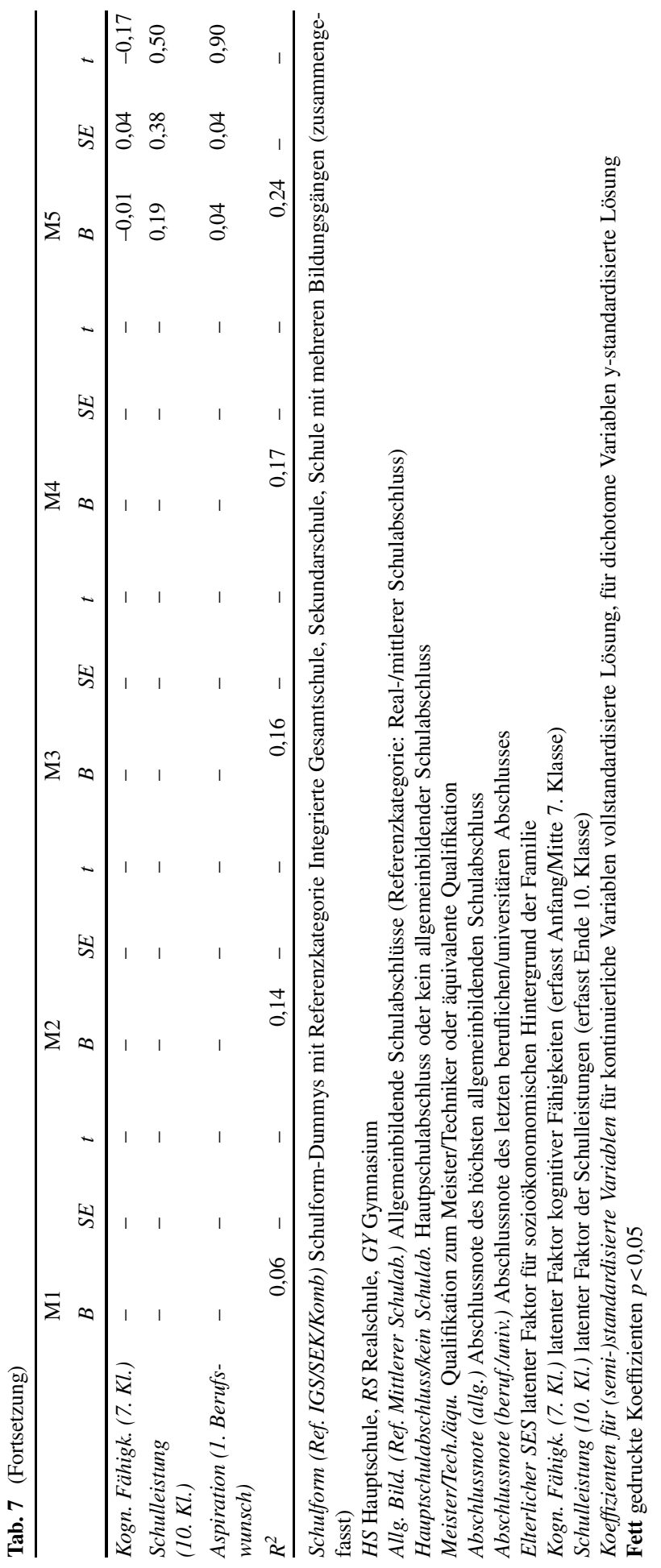




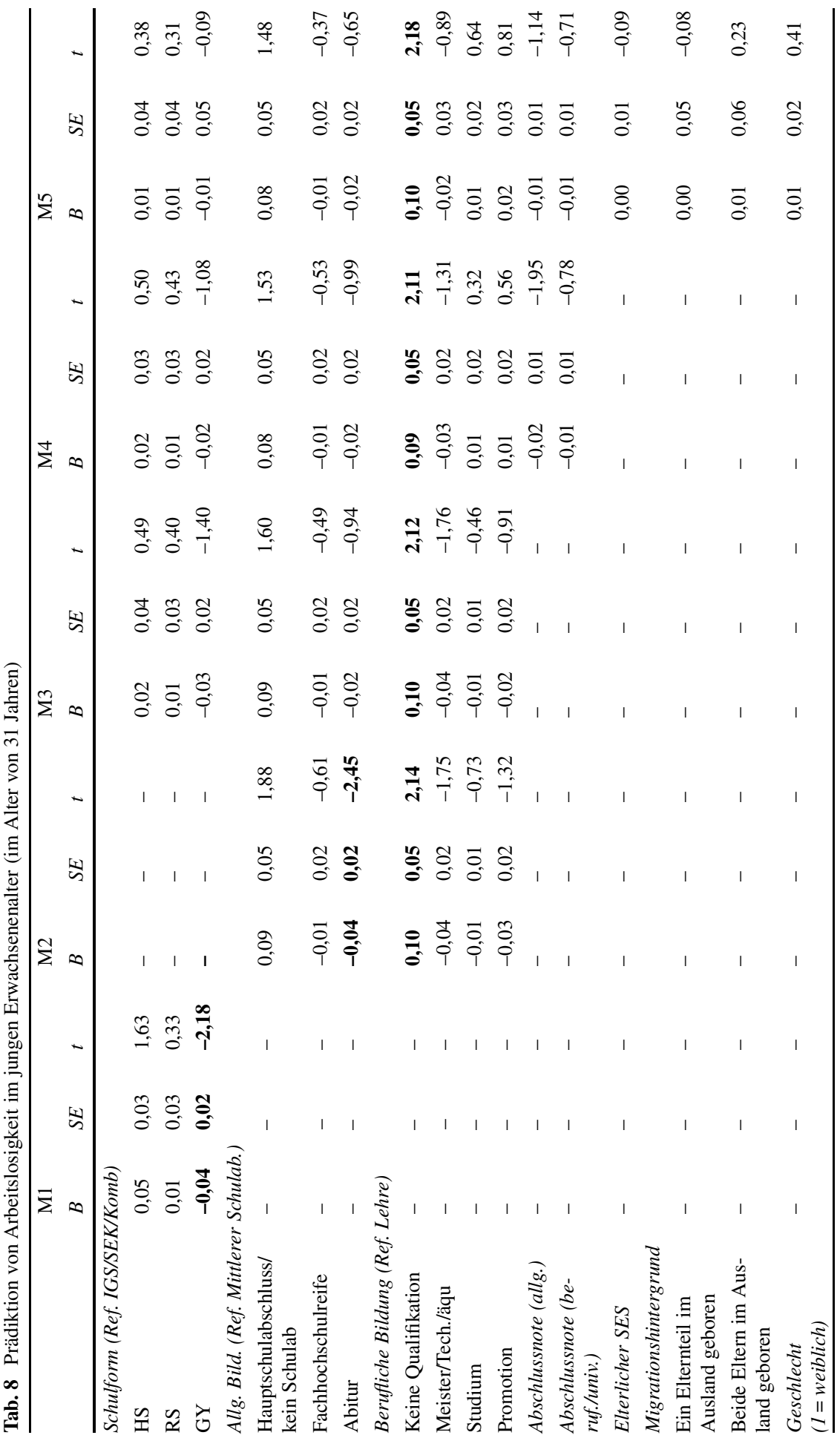




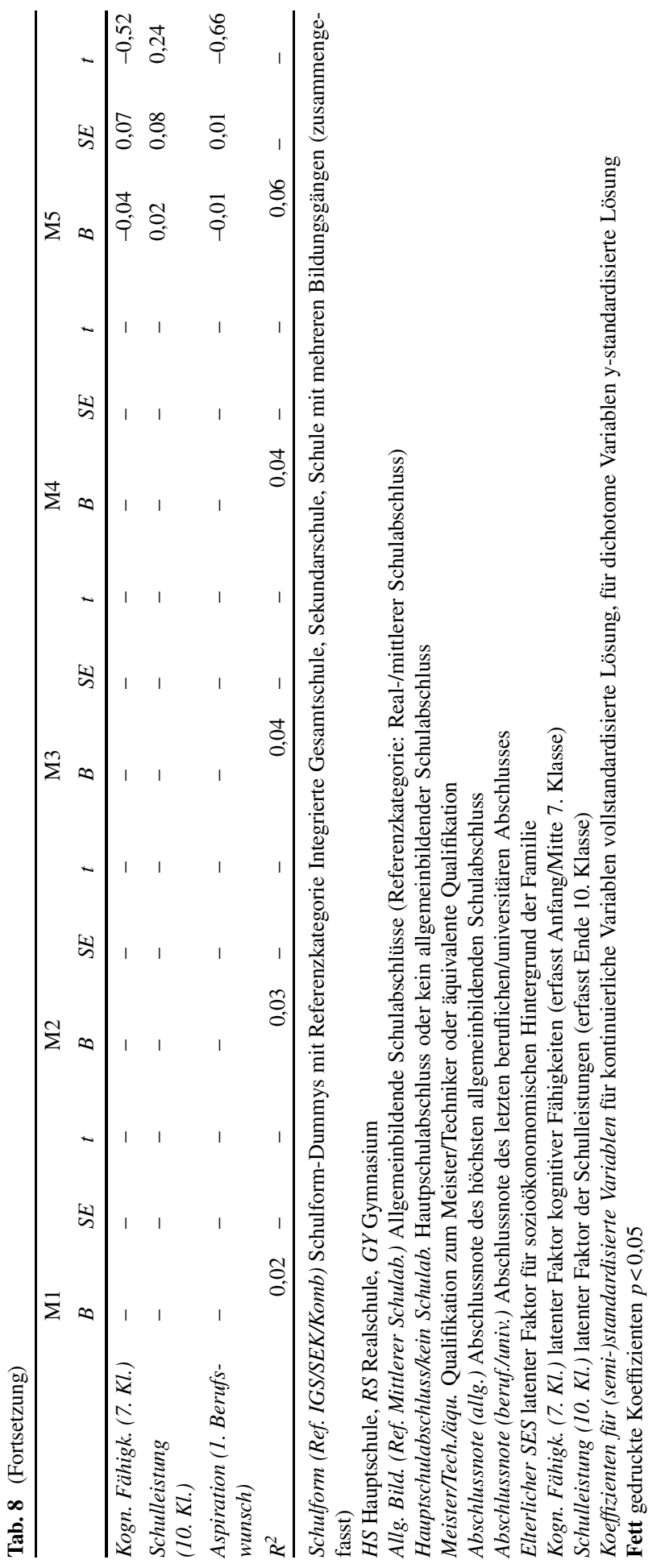


Wenngleich sich für die meisten Variablen eine korrelative Beziehung mit Arbeitslosigkeit findet (vgl. Tab. 5), erwiesen sich die Effekte in der multivariaten Analyse stark konfundiert (vgl. Tab. 8). Für die Vorhersage von Arbeitslosigkeit traten in den Modellen M1 und M2 statistisch bedeutsame Effekte sowohl für die besuchte Schulform in der Sekundarstufe (M1) - hier aber beschränkt auf den Besuch eines Gymnasiums - als auch für das Abitur und vor allem für das Fehlen eines Abschlusses (M2) auf. In einer gemeinsamen Modellierung im Modell M3 blieb allerdings lediglich das Fehlen eines Abschlusses statistisch bedeutsam (die Schulnote verfehlt knapp statistische Bedeutsamkeit). Dies wiederholt sich auch in den Modellen M4 und M5. Ausbildungslosigkeit scheint im jungen Erwachsenenalter der dominante Prädiktor von Arbeitslosigkeit zu sein.

\section{Zusammenfassung und Diskussion}

Gegenstand des vorliegenden Beitrags war die Frage, inwiefern die besuchte Schulform im Jugendalter den sozioökonomischen Erfolg im jungen Erwachsenenalter (im Alter von 31 Jahren) auch dann noch vorhersagt, wenn zugleich die erreichte schulische und berufliche Qualifikation (sowie weitere individuelle und soziale Hintergrundvariablen) kontrolliert werden. Zusammenfassend lässt sich konstatieren, dass sich partiell sehr deutliche Unterschiede zwischen den besuchten Schulformen in Hinblick auf den beruflichen Status, das Einkommen und Arbeitslosigkeit im jungen Erwachsenenalter identifizieren ließen. Im Wesentlichen zeigten sich diese Unterschiede stark mit den erreichten schulischen und beruflichen Qualifikationen konfundiert, die unterschiedlich häufig in den spezifischen Schulformen erworben wurden. Andererseits fiel diese Konfundierung differenziell je nach betrachteter Dimension des soziö̈konomischen Erfolges aus: In Hinblick auf den beruflichen Status verloren Unterschiede zwischen Schulformen ihre statistische Bedeutung erst dann, wenn zusätzlich die Abschlussnoten und individuelle kognitive, motivationale sowie soziographische Unterschiede kontrolliert wurden. Ausgenommen hiervon ist allerdings die Hauptschule: Mit dem Besuch einer Hauptschule geht auch unter Berücksichtigung der anderen strukturellen und individuellen Hintergrundvariablen ein niedrigerer beruflicher Status einher. Für das Einkommen hingegen verschwanden Unterschiede zwischen den ehemals besuchten Schulformen unmittelbar, wenn die schulischen und beruflichen Abschlüsse kontrolliert wurden. Diese Effekte unterschiedlicher schulischer und beruflicher Abschlüsse erwiesen sich auch bei Kontrolle des individuellen und sozialen Hintergrunds robust. In diesem Sinne sind die Befunde zur Arbeitslosigkeit konsistent, da vor allem Ausbildungslosigkeit Arbeitslosigkeit vorhersagt, weniger der Besuch unterschiedlicher Schulformen in der Sekundarstufe.

Diese Befunde stehen weitgehend, aber auch nicht vollständig, im Einklang mit der vorangehenden Literatur und ergänzen und erweitern sie in verschiedener Hinsicht. Einerseits bestätigen die Ergebnisse die Befunde von Fend (2009a, b, 2014), der im Rahmen der LifE-Studie keine langfristigen Effekte der Schulstrukturen aus der Sekundarstufe fand: Die in der Sekundarstufe identifizierten günstigeren meritokratischen Effektmuster in ungegliederten Systemen schienen sich langfristig im 
(beruflichen) Erwachsenenalter zu verlieren. Im vorliegenden Fall und mit dem Fokus auf die Fragestellung der Schulformeffekte innerhalb eines Systems zeigte sich ein ähnlicher Befund. Vor allem für die mittleren Schulformen, d.h. Real- und unterschiedliche Gesamtschulformen, und die Gymnasien finden sich im Hinblick auf beide betrachteten Ergebnisvariablen keine weiteren Effekte, wenn die tatsächlich erworbenen Abschlüsse einbezogen werden. Für die Hauptschulen gilt dies auch für das Einkommen und die Arbeitslosigkeit, für das berufliche Prestige allerdings nicht. Hier bleiben Effekte des Besuchs einer Hauptschule bestehen, auch wenn der individuelle Hintergrund berücksichtigt wird.

Ähnlich stellt sich dies für die Ergebnisse aus Dumont et al. (2017) dar: Wie Jugendliche ihre beruflichen Chancen einschätzen, wird maßgeblich durch Effekte der Abschlüsse und nicht der besuchten Schulformen vorhergesagt, was dem hier berichteten Effektmuster nur teilweise entspricht. Gleichzeitig berichteten die Autorinnen und Autoren auch, dass sich die typischen positiven Kontexteffekte im Sinne von Big Fish Little Pond-Effekten (BFLPE) des schulischen Kontextes auf die akademischen Selbstkonzepte nachweisen lassen. Diese wurden in dieser Literatur als protektive Wirkungsmechanismen von Schulformen für leistungsschwächere Schülerinnen und Schüler diskutiert, die sich dann längerfristig positiv auf die Entwicklungen auswirken könnten (im Sinne selbstwertschützender Nischen; vgl. Marsh et al. 2008). Diese Sichtweise wird durch die hier vorliegenden Analysen nicht unterstützt, da die Schulformen weitgehend neutrale, im kritischen Fall der Hauptschule aber eher negative Effekte auf den beruflichen Status zeigten; Hinweise auf positive Effekte der weniger leistungsstarken schulischen Milieus deuten sich nicht an. Zumindest partiell unterstützen unsere Befunde die an anderer Stelle geäußerten Vermutungen negativer Effekte der Hauptschule (vgl. Knigge und Hannover 2011; Solga und Wagner 2001).

Unsere Ergebnisse schließen mit Einschränkungen auch an diejenigen Arbeiten an, die querschnittlich auf Basis der NEPS-Daten die Zugangswege für das Abitur und Fachabitur untersuchten (Schuchart und Rürup 2017; Schuchart und Schimke 2019; Sterrenberg 2014) und erweitern diese. In diesen drei Arbeiten erwiesen sich die Abschlusszertifikate als die zentralen Prädiktoren. Schulformen waren darüber hinaus kaum prädiktiv, aber es fand sich ein negativer Effekt für die Interaktion zwischen Alter und Schulform, spezifisch: Für das späte Nachholen eines Schulabschlusses (mit 25 Jahren oder älter). Das Alter wiederum wurde in der vorliegenden Arbeit nicht weiter differenziert, da die Studie aufgrund des jungen Erwachsenenalters (von knapp 31 Jahren) in der letzten Erhebung noch relativ nahe an dieser Altersgrenze liegt. Entsprechend wäre diese Interaktion zwischen Schulform und Alter derjenige Aspekt, der am stärksten weiterer empirischer Prüfung in längsschnittlichen Designs bedarf.

Allerdings verbleibt auch ein Effekt des elterlichen sozioökonomischen Hintergrundes auf das spätere berufliche Prestige (siehe auch Becker et al. 2019): Analog zu den Effekten des Hauptschulbesuches findet sich ein Effekt des elterlichen sozioökonomischen Hintergrunds auf das berufliche Prestige. Dieses Muster lässt sich dahingehend zusammenfassen, dass beide Effekte mit der Wahl statusärmerer Berufe einhergehen, die aber nicht unbedingt schlechter entlohnt werden und auch in Hinblick auf das Arbeitslosigkeitsrisiko neutral erscheinen. Anders hingegen ver- 
hält es sich mit Unterschieden zwischen den Geschlechtern. Diese treten gerade für das Einkommen und nicht für das Berufsprestige oder Arbeitslosigkeit auf (vgl. für vertiefende Analysen Becker et al. 2019). Keine Unterschiede finden sich für die familiäre Migrationsgeschichte, was zwar auch in Übereinstimmung mit den Befundmustern in NEPS steht (Schuchart und Rürup 2017), aber möglicherweise (in beiden Studien) mit Vorsicht zu interpretieren ist, da hier aufgrund der geringen Zellenbesetzung die Indikatoren nicht so weit ausdifferenziert sind, wie dies notwendig wäre (z. B. nach Herkunftsgruppen und Generationenstatus).

Hinsichtlich der allgemeinen oder spezifischen Qualifikationen findet sich eine klare Dominanz der beruflichen Bildung. Berufliche Bildung zeigte sich in allen Modellen als das zentrale Prädiktorenbündel. Effekte der allgemeinbildenden Abschlusszertifikate blieben weitgehend auf das (Fach-)Abitur beschränkt, zumindest bei der Vorhersage des beruflichen Status. Möglicherweise ist dies Resultat der Optionslogik nach ,unten“, also dass auch Leute mit Abitur Berufe ergreifen, die kein solches voraussetzen. Hiervon mag möglicherweise eine positive Signalwirkung des Abiturs auf dem Arbeitsmarkt ausgehen, was aber im Rahmen des hier eingesetzten Designs nicht abschließend zu beurteilen ist.

Grundlegend ist bei der Interpretation der Effekte der Schulformen von Bedeutung, dass sich die hier vorgelegten Befunde maßgeblich auf Effekte der alternativen Wege in den Arbeitsmarkt beziehen. Eine andere Frage ist, in welchem Zusammenhang die Schulformen mit den unmittelbaren allgemeinbildenden und beruflichen Abschlüssen selbst stehen. Dies ist eine Fragestellung, die wir hier nicht explizit behandelt haben. Dass den Schulformen in diesen vorgelagerten Stadien durchaus eine Bedeutung zukommt, deutet sich etwa darin an, dass sich eine mittlere bis starke Korrelation zwischen den besuchten Schulformen und den später erzielten Abschlüssen findet (siehe Tab. 3 und 4). Dieser Zusammenhang bleibt auch bestehen, wenn man z. B. die hier verwendeten Hintergrundvariablen einbezieht (vgl. auch Anhang, Tab. 9). Insofern lässt sich ein differenzielles Effektmuster zwischen einerseits den Ausbildungswegen und andererseits den Erträgen von Schulformen vs. Zertifikaten auf dem Arbeitsmarkt ausmachen. Somit scheint es gerechtfertigt, davon auszugehen, dass Schulformen auch indirekt mit weiterer Heterogenität zwischen Personen assoziiert sind (vgl. auch Protsch 2014; Solga und Wagner 2001). Allerdings wird dies zumindest nicht direkt über differenzielle Lernverläufe in den jeweiligen Schulformen vermittelt: Auch wenn man kognitive und psychosoziale Faktoren, die durch die Schulformen beeinflusst sein könnten, aus den Modellen ausschließt, zeigte sich das gleiche Befundmuster (vgl. Anhang, Tab. 10).

\subsection{Methodische Implikationen und Einschränkungen der Arbeit}

Trotz der methodischen Vorteile, die diese Untersuchung aufweist, vor allem aufgrund der längsschnittlichen Anlage und der Möglichkeit, Schulform und Zertifikat eindeutig zu differenzieren, sowie soziale, kognitive und motivationale Hintergrundvariablen aus der Jugendzeit zu kontrollieren, ergeben sich auch eine Reihe von Einschränkungen. Zunächst ist zu beachten, dass diese Studie lediglich 4 Bundesländer (Berlin, Mecklenburg-Vorpommern, Sachsen-Anhalt und Nordrhein-Westfalen) erfasste und damit nicht repräsentativ auf nationaler Ebene ist. Zudem wurde 
lediglich eine historische Kohorte, die 1991/92 die 7. Klasse besuchte, untersucht. Inwieweit die Ergebnisse auf Bundesebene und auf andere historische Kohorten generalisierbar sind, ist eine offene Frage, wenngleich die ähnlichen Befundmuster aus der national repräsentativ gezogenen (aber querschnittlichen) NEPS-Studie für eine relativ robuste Generalisierbarkeit sprächen.

Bei der Interpretation der Ergebnisse gilt ferner zu berücksichtigen, dass Erwachsene im Alter von etwas mehr als 30 Jahren betrachtet werden, die berufliche Entwicklung in diesem Alter aber noch nicht für alle Personen abgeschlossen ist. Längsschnittliche Untersuchungen aus anderen Ländern (vgl. z. B. für die USA Ganzach 2011; Spengler et al. 2018; Zax und Rees 2002) und auch querschnittlichen Analysen etwa auf Basis der PIAAC-Daten (Hanushek et al. 2015) legen zwar nahe, dass die Zusammenhänge (nicht unbedingt die Niveau-Unterschiede) nach dem Alter von 30 Jahren im Wesentlichen stabil bleiben (Strenze 2007). Dies bezieht sich allerdings maßgeblich auf historisch ältere Kohorten, und somit obliegt es wiederum späterer Prüfung, ob dies auch auf jüngere Kohorten und deren partiell verzögerte Lebensverläufe (Settersten und Ray 2010) generalisierbar ist. Spezifisch wurde für das Arbeitslosigkeitsrisiko berichtet, dass sich z. B. im jüngeren und mittleren Erwachsenenalter verschiedene Ausbildungsgruppen wenig unterscheiden und erst in der späten Karriere Unterschiede vor allem zugunsten von Akademikerinnen und Akademikern auftreten (Forster et al. 2016). Insofern erscheint es als noch offene und deshalb wichtige Frage, ob bzw. wie sich die hier gefundenen Effekte im weiteren Lebensverlauf und der weiteren beruflichen Karriere noch verändern.

In Hinblick auf die Robustheit unserer Analysen haben wir zudem die Stabilität unserer Operationalisierungen überprüft. Wenngleich das Dropout-Muster demjenigen vergleichbarer Studien ähnelt (z.B. Damian et al. 2015; Fend et al. 2009; Schoon 2008; Spengler et al. 2015; von Stumm et al. 2010), bleibt das Problem (all dieser Studien) bestehen, dass der Dropout differenziell ausfällt und vor allem Personen mit geringeren kognitiven und sozialen Ressourcen aus der Studie ausscheiden (vgl. Tab. 2). Dies ist dahingehend bedeutsam, weil wir mit der Prüfung der Effekte bezüglich der Hauptschulen auch an Unterschieden gerade in diesen Bereichen der Verteilungen, also den kognitiv weniger starken und sozial weniger privilegierten Schülerinnen und Schüler interessiert waren. Insgesamt betrachtet erwiesen sich die Ergebnismuster auch robust in inklusiveren Stichprobenspezifikationen (z.B. mindestens einmal ab Welle 4/Klasse 10 teilgenommen, $N=10.827$ ). Ähnliche Ergebnismuster zeigten sich auch, wenn lediglich diejenigen Bundesländer einbezogen wurden, in denen die Hauptschule generell 10-jährig geführt wurde (d.h. NRW und Berlin-West; vgl. Statistisches Bundesamt 1993). Allerdings sind auch diese Zusatzanalysen aufgrund des differenziellen Datenausfalls in Hinblick auf weniger leistungsstarke Schülerinnen und Schüler und solche aus sozial weniger privilegierten Elternhäusern nur unter Vorbehalt zu interpretieren, da zumindest die Grundannahme der Modelle gegeben sein muss (d. h. Werte dürfen nur (conditionally) at random und nicht systematisch fehlen; vgl. Lüdtke und Robitzsch 2011). Dies bleibt aber stets eine Annahme und kann nicht getestet werden. Die hier berichteten Befunde sind insofern eher als konservative Effekte anzusehen, und die wahren Effekte könnten noch größer sein. Vor allem in Hinblick auf die Vorhersage von Arbeitslosigkeit erscheint dies bedeutsam, da das an sich schon relativ seltene Ereignis 
durch die positive Selektivität der Stichprobe (noch) weniger robust vorhersagbar sein sollte. Insofern sind gerade hier die vorliegenden Ergebnisse nur mit Vorsicht zu interpretieren. Entsprechend sind weitere Replikationen mit anderen Daten, und vielleicht insbesondere mit querschnittlichen Daten, die weniger von dieser Art der längsschnittlichen Selektivität betroffen sind, indiziert, um die Belastbarkeit der hier vorgestellten Befunde zu prüfen.

Darüber hinaus haben wir die Stabilität der Ergebnisse auch in Hinblick auf die Konstruktoperationalisierungen überprüft. Zentral sind hierbei vor allem alternative Operationalisierungen einerseits von Bildung (als Bildung in Jahren gemeinsam oder getrennt für allgemeine und berufliche Bildung), andererseits der kognitiven Leistungsfähigkeit (nur Intelligenz zur 7. Klasse bzw. nur Schulleistung zur 10./7. Klasse; vgl. auch Anhang, Tab. 10). Auch haben wir die Ergebnisse mit weniger sparsamen, manifesten Modellen bezüglich der Hintergrundvariablen (elterlicher sozioökonomischer Hintergrund, kognitive Grundfähigkeiten und Schulleistungen) und zusätzlich mit der Dauer der Berufserfahrung als weiteren Prädiktor getestet. Im Wesentlichen zeigte sich mit diesen Varianten ein nahezu identisches Effektmuster. Nicht zuletzt zeigte sich in Hinblick auf Schulformeffekte ein weitgehend identisches Befundmuster, wenn die Analysen nach Schulabschluss getrennt (Hauptschulabschluss und mittlerer Schulabschluss sowie (Fach-)Abitur) durchgeführt wurden.

\subsection{Ausblick}

Mit der vorliegenden Arbeit wurde erstmals eine Analyse vorgelegt, die längsschnittlich die differenzielle Bedeutsamkeit der unterschiedlichen Schulformen über die Lebensspanne, von der Kindheit in das junge Erwachsenenalter hinein in Hinblick auf sozioökonomische berufliche Erträge zu untersuchen erlaubte. Grundlegend liefert die vorliegende Arbeit Hinweise darauf, dass die Entscheidung für die eine oder andere Schulform am Ende der Grundschulzeit auch direkte Folgen auf dem Arbeitsmarkt haben kann - so der Besuch einer Hauptschule in der Sekundarschulzeit auf das erzielte berufliche Prestige im jungen Erwachsenenalter. Dies ist auch aus der Perspektive der Verteilungsgerechtigkeit von Bedeutung, da die Verteilung auf die Schulformen auch durch soziale Aspekte mitbeeinflusst wird. Darüber hinaus weisen die Analysen auf weitere problematische Effekte hin, so etwa der direkte Einfluss des sozialen Hintergrundes auf das berufliche Prestige, Geschlechtereffekte auf das Einkommen, aber auch indirekte Effekte der Schulformen und der sozialen Herkunft auf Abschlusszertifikate (vgl. auch Becker et al. 2019; sowie Anhang, Tab. 10). Aber dies ist auf andere Prozesse als auf direkte Auswirkungen von Schulformen zurückzuführen (vgl. auch Fend 2009a, b, 2014). Hier müssten künftige Studien anschließen, die näher über die Mechanismen der direkten (und indirekten) Effekte von Schulformen Aufschluss geben können, ob beispielsweise mit dem Besuch einer Hauptschule Berufspräferenzen geprägt werden und hierdurch Berufswahlen in Hinblick auf das berufliche Prestige konservativer ausfallen, oder ob ehemalige Hauptschülerinnen und Hauptschüler in prestigereichen Berufen besondere Hürden zu nehmen haben und eine institutionelle Diskriminierung erfahren. In diesem Kontext ist weiterführend von Bedeutung, dass sich das deutsche Schulsystem in den letzten Jahren in Hinblick auf die Sekundarschulstrukturen weiter 
verändert hat - gerade die Anschlussoptionen wurden zugunsten einer weiteren Flexibilisierung und Offenheit gestärkt (Hurrelmann 2013; Neumann et al. 2017a, b, 2013; Tillmann 2012). Entsprechend wird in den kommenden Jahren zu sehen sein, ob die weitere institutionelle Flexibilisierung $\mathrm{zu}$ weiteren ausgleichenden Effekten in höherem Alter und vor allem in nachfolgenden Kohorten führen wird.

Danksagung Wir danken Mona Frermann, Jan Scharf, Gerrit Hasche, Annika Koch und Susannah Goss für hilfreiche Kommentare und die Unterstützung bei der Manuskripterstellung.

Funding Open Access funding enabled and organized by Projekt DEAL.

Open Access Dieser Artikel wird unter der Creative Commons Namensnennung 4.0 International Lizenz veröffentlicht, welche die Nutzung, Vervielfältigung, Bearbeitung, Verbreitung und Wiedergabe in jeglichem Medium und Format erlaubt, sofern Sie den/die ursprünglichen Autor(en) und die Quelle ordnungsgemäß nennen, einen Link zur Creative Commons Lizenz beifügen und angeben, ob Änderungen vorgenommen wurden.

Die in diesem Artikel enthaltenen Bilder und sonstiges Drittmaterial unterliegen ebenfalls der genannten Creative Commons Lizenz, sofern sich aus der Abbildungslegende nichts anderes ergibt. Sofern das betreffende Material nicht unter der genannten Creative Commons Lizenz steht und die betreffende Handlung nicht nach gesetzlichen Vorschriften erlaubt ist, ist für die oben aufgeführten Weiterverwendungen des Materials die Einwilligung des jeweiligen Rechteinhabers einzuholen.

Weitere Details zur Lizenz entnehmen Sie bitte der Lizenzinformation auf http://creativecommons.org/ licenses/by/4.0/deed.de. 


\section{Anhang}

Tab. 9 Prädiktion von Qualifikationen im jungen Erwachsenenalter (im Alter von 31 Jahren): Allgemeinbildender Schulabschluss (in Jahren) sowie allgemeinbildender und beruflicher Abschluss (CASMIN-Kodierung in Jahren)

\begin{tabular}{|c|c|c|c|c|c|c|}
\hline & \multicolumn{3}{|c|}{$\begin{array}{l}\text { Allgemeinbildende Schulabschlüsse } \\
\text { (in Jahren) }\end{array}$} & \multicolumn{3}{|c|}{$\begin{array}{l}\text { Allg. u. berufliche Bild. } \\
\text { (CASMIN, in Jahren) }\end{array}$} \\
\hline & $B$ & $S E$ & $t$ & $B$ & $S E$ & $t$ \\
\hline \multicolumn{7}{|c|}{ Schulform (Ref. IGS/SEK/Komb.) } \\
\hline HS & $-0,48$ & $\mathbf{0 , 1 0}$ & $-4,93$ & $-0,31$ & $\mathbf{0 , 1 0}$ & $-3,10$ \\
\hline RS & $-0,08$ & 0,10 & $-0,81$ & $-0,02$ & 0,08 & $-0,27$ \\
\hline GY & 0,68 & 0,16 & 4,37 & $\mathbf{0 , 3 9}$ & $\mathbf{0 , 1 3}$ & 3,10 \\
\hline Elterlicher SES & $\mathbf{0 , 1 0}$ & $\mathbf{0 , 0 3}$ & 3,66 & $\mathbf{0 , 2 1}$ & $\mathbf{0 , 0 3}$ & $\mathbf{7 , 5 3}$ \\
\hline \multicolumn{7}{|c|}{ Migrationshintergrund (Ref.: Eltern in D geboren) } \\
\hline $\begin{array}{l}\text { Ein Elternteil im } \\
\text { Ausland geboren }\end{array}$ & $-0,03$ & 0,09 & $-0,34$ & $-0,03$ & 0,11 & $-0,24$ \\
\hline $\begin{array}{l}\text { Beide Elternteile im } \\
\text { Ausland geboren }\end{array}$ & 0,20 & 0,14 & 1,42 & 0,23 & 0,13 & 1,73 \\
\hline $\begin{array}{l}\text { Geschlecht } \\
(1=\text { weiblich })\end{array}$ & $-0,04$ & 0,05 & $-0,81$ & $-0,07$ & 0,05 & $-1,54$ \\
\hline $\begin{array}{l}\text { Kogn. Fähigk. } \\
\text { (7. Kl.) }\end{array}$ & 0,25 & 0,07 & 3,63 & 0,34 & 0,06 & $\mathbf{5 , 5 5}$ \\
\hline $\begin{array}{l}\text { Aspiration (1. Be- } \\
\text { rufswunsch) }\end{array}$ & 0,14 & 0,04 & 3,78 & 0,11 & $\mathbf{0 , 0 3}$ & 3,54 \\
\hline$R^{2}$ & 0,48 & - & - & 0,50 & - & - \\
\hline
\end{tabular}

Allg. Bild. (in Jahren) Höchster allgemeinbildender Schulabschluss (in Jahren)

Allg. u. berufliche Bild. (CASMIN, in Jahren) allgemeinbildender und beruflicher Abschluss zusammengefasst in Jahren als CASMIN-Kodierung

Schulform (Ref. IGS/SEK/Komb.) Schulform-Dummys mit Referenzkategorie Integrierte Gesamtschule, Sekundarschule, Schule mit mehreren Bildungsgängen (zusammengefasst)

HS Hauptschule, RS Realschule, GY Gymnasium

Elterlicher SES latenter Faktor für sozioökonomomischen Hintergrund der Familie

Kogn. Fähigk. (7. Kl.) latenter Faktor kognitiver Grundfähigkeiten (erfasst 7. Klasse)

Koeffizienten für (semi-)standardisierte Variablen für kontinuierliche Variablen vollstandardisierte Lösung, für dichotome Variablen y-standardisierte Lösung

FIML-basierte Analysen

Fett gedruckte Koeffizienten $p<0,05$ 
Tab. 10 Reduziertes Modell der Prädiktion des beruflichen Status, Einkommens (logarithmiert) und Erwerbslosigkeit durch Schulformbesuch, allgemeinbildende und berufliche Abschlusszertifikate sowie familiären und frühem individuellen Hintergrund (kognitive Grundfähigkeiten zu Beginn der Klasse 7)

\begin{tabular}{|c|c|c|c|c|c|c|c|c|c|}
\hline & \multicolumn{2}{|l|}{ ISEI } & \multicolumn{4}{|c|}{ Einkommen } & \multicolumn{3}{|c|}{ Arbeitslosigkeit } \\
\hline & $B$ & $S E$ & $t$ & $B$ & $S E$ & $t$ & $B$ & $S E$ & $t$ \\
\hline \multicolumn{10}{|c|}{ Schulform (Ref. IGS/SEK/Komb) } \\
\hline HS & $-0,24$ & $\mathbf{0 , 0 8}$ & $-3,06$ & 0,02 & 0,12 & 0,21 & 0,01 & 0,03 & 0,33 \\
\hline RS & $-0,01$ & 0,07 & $-0,14$ & 0,09 & 0,09 & 1,03 & 0,02 & 0,03 & 0,65 \\
\hline GY & 0,00 & 0,10 & 0,01 & 0,13 & 0,11 & 1,19 & 0,01 & 0,03 & 0,20 \\
\hline \multicolumn{10}{|c|}{ Allg. Bild. (Ref. Mittl. Schulab.) } \\
\hline $\begin{array}{l}\text { Hauptschulab./kein } \\
\text { Schulab }\end{array}$ & $-0,14$ & 0,10 & $-1,35$ & $-0,16$ & 0,18 & $-0,92$ & 0,08 & 0,05 & 1,47 \\
\hline Fachhochschulreife & $\mathbf{0 , 1 8}$ & $\mathbf{0 , 0 7}$ & 2,71 & 0,13 & 0,09 & 1,44 & $-0,01$ & 0,02 & $-0,41$ \\
\hline Abitur & $\mathbf{0 , 3 7}$ & $\mathbf{0 , 0 8}$ & 4,44 & 0,16 & 0,09 & 1,72 & $-0,02$ & 0,02 & $-0,68$ \\
\hline \multicolumn{10}{|c|}{ Berufliche Bildung (Ref. Lehre) } \\
\hline $\begin{array}{l}\text { Keine Qualifikati- } \\
\text { on }\end{array}$ & $-0,06$ & 0,11 & $-0,53$ & $-0,75$ & $\mathbf{0 , 1 7}$ & $-4,52$ & $\mathbf{0 , 1 0}$ & $\mathbf{0 , 0 5}$ & 2,26 \\
\hline Meister/Tech./äqu & 0,09 & 0,07 & 1,25 & 0,25 & 0,09 & 2,97 & $-0,03$ & 0,02 & $-1,19$ \\
\hline Studium & $\mathbf{0 , 8 0}$ & 0,06 & 13,72 & 0,38 & $\mathbf{0 , 0 7}$ & 5,13 & 0,01 & 0,02 & 0,75 \\
\hline Promotion & 1,09 & 0,09 & 11,94 & 0,60 & $\mathbf{0 , 1 1}$ & 5,37 & 0,02 & 0,02 & 0,90 \\
\hline $\begin{array}{l}\text { Abschlussnote } \\
\text { (allg.) }\end{array}$ & 0,06 & $\mathbf{0 , 0 3}$ & 2,39 & 0,12 & 0,04 & 3,32 & $-0,01$ & 0,01 & $-1,17$ \\
\hline $\begin{array}{l}\text { Abschlussnote } \\
\text { (beruf./univ.) }\end{array}$ & $-0,01$ & 0,03 & $-0,37$ & $-0,02$ & 0,03 & $-0,57$ & $-0,01$ & 0,01 & $-0,70$ \\
\hline Elterlicher SES & $\mathbf{0 , 1 0}$ & $\mathbf{0 , 0 3}$ & $\mathbf{3 , 0 3}$ & 0,00 & 0,04 & $-0,09$ & 0,00 & 0,01 & $-0,09$ \\
\hline \multicolumn{10}{|c|}{ Migrationshintergrund } \\
\hline $\begin{array}{l}\text { Ein Elternteil im } \\
\text { Ausland geb }\end{array}$ & $-0,11$ & 0,12 & $-0,90$ & 0,28 & 0,18 & 1,60 & 0,00 & 0,05 & $-0,08$ \\
\hline $\begin{array}{l}\text { Beide Eltern im } \\
\text { Ausland geb }\end{array}$ & $-0,12$ & 0,13 & $-0,87$ & 0,17 & 0,19 & 0,92 & 0,01 & 0,05 & 0,26 \\
\hline $\begin{array}{l}\text { Geschlecht } \\
(1=\text { weiblich })\end{array}$ & 0,05 & 0,04 & 1,10 & $-0,46$ & 0,05 & $-9,36$ & 0,01 & 0,01 & 0,49 \\
\hline $\begin{array}{l}\text { Kogn. Fähigk. } \\
\text { (7. Kl.) }\end{array}$ & 0,11 & 0,06 & 1,87 & 0,01 & 0,07 & 0,16 & $-0,02$ & 0,02 & $-1,10$ \\
\hline$R^{2}$ & 0,50 & - & - & 0,24 & - & - & 0,07 & - & - \\
\hline
\end{tabular}

Schulform (Ref. IGS/SEK/Komb) Schulform-Dummys mit Referenzkategorie Integrierte Gesamtschule, Sekundarschule, Schule mit mehreren Bildungsgängen (zusammengefasst)

$H S$ Hauptschule, RS Realschule, GY Gymnasium

Allg. Bild. (Ref. Mittl. Schulab.) Allgemeinbildende Schulabschlüsse (Referenzkategorie: Real-/mittlerer Schulabschluss)

Hauptschulab./kein Schulab. Hautpschulabschluss oder kein allgemeinbildender Schulabschluss Meister/Tech./äqu. Qualifikation zum Meister/Techniker oder äquivalente Qualifikation Abschlussnote (allg.) Abschlussnote des höchsten allgemeinbildenden Schulabschluss Abschlussnote (beruf./univ.) Abschlussnote des letzten beruflichen/universitären Abschlusses Elterlicher SES latenter Faktor für sozioökonomomischen Hintergrund der Familie

Kogn. Fähigk. (7. Kl.) latenter Faktor kognitiver Fähigkeiten (erfasst Anfang/Mitte 7. Klasse)

Koeffizienten für (semi-)standardisierte Variablen für kontinuierliche Variablen vollstandardisierte Lösung, für dichotome Variablen y-standardisierte Lösung

Fett gedruckte Koeffizienten $p<0,05$ 


\section{Literatur}

Amthauer, R. (1955). I-S-T. Intelligenz-Struktur-Test: Handanweisung für die Durchführung und Auswertung (2. Aufl.). Göttingen: Hogrefe.

Amthauer, R., Brocke, B., Liepmann, D., \& Beauducel, A. (2001). Intelligenz-Struktur-Test 2000R. Göttingen: Hogrefe.

Autorengruppe Bildungsberichterstattung (Hrsg.). (2018). Bildung in Deutschland 2018: Ein indikatorengestützter Bericht mit einer Analyse zu Wirkungen und Erträgen von Bildung. Bielefeld: wbv.

Bachsleitner, A., Becker, M., Neumann, M., \& Maaz, K. (2018). Social background effects in the transition to a doctoral degree: empirical evidence from a German longitudinal study. Research in Social Stratification and Mobility. https://doi.org/10.1016/j.rssm.2018.07.004.

Baethke, M. (2008). Das berufliche Bildungswesen in Deutschland am Beginn des 21. Jahrhunderts. In K. S. Cortina, J. Baumert, A. Leschinsky, K. U. Mayer \& L. Trommer (Hrsg.), Das Bildungswesen in der Bundesrepublik Deutschland: Strukturen und Entwicklungen im Überblick (S. 541-597). Reinbeck bei Hamburg: Rowohlt.

Baumert, J., Lehmann, R., Lehrke, M., Schmitz, B., Clausen, M., Hosenfeld, I., \& Neubrand, J. (1997). TIMSS: Mathematisch-naturwissenschaftlicher Unterricht im internationalen Vergleich: Deskriptive Befunde. Opladen: Leske + Budrich.

Baumert, J., Cortina, K. S., \& Leschinsky, A. (2008). Grundlegende Entwicklungen und Strukturprobleme im allgemeinbildenden Schulwesen. In K. S. Cortina, J. Baumert, A. Leschinsky, K. U. Mayer \& L. Trommer (Hrsg.), Das Bildungswesen in der Bundesrepublik Deutschland: Strukturen und Entwicklungen im Überblick (S. 53-130). Reinbeck bei Hamburg: Rowohlt.

Baumert, J., Maaz, K., Neumann, M., Becker, M., \& Dumont, H. (2017). Die Berliner Schulstrukturreform: Hintergründe, Zielstellungen und theoretischer Rahmen. In M. Neumann, M. Becker, J. Baumert, K. Maaz \& O. Köller (Hrsg.), Zweigliedrigkeit im deutschen Schulsystem: Potenziale und Herausforderungen in Berlin (S. 9-38). Münster: Waxmann.

Baumert, J., Dumont, H., Becker, M., Neumann, M., Bachsleitner, A., Köller, O., \& Maaz, K. (2018). Soziokulturelle und geschlechtsspezifische Selektivität von Übergangsberechtigungen für die gymnasiale Oberstufe in mehr-und zweigliedrigen Schulsystemen. Kölner Zeitschrift für Soziologie und Sozialpsychologie, 70(4), 593-628. https://doi.org/10.1007/s11577-018-0584-0.

Baumert, J., Neumann, M., Dumont, H., Becker, M., Bachsleitner, A., Maaz, K., \& Köller, O. (2019). Platzierungsentscheidungen beim Übergang in die gymnasiale Oberstufe: Folgen der Umstellung auf Zweigliedrigkeit des Sekundarschulsystems in Berlin. Zeitschrift für Erziehungswissenschaft, 22(4), 721-763. https://doi.org/10.1007/s11618-019-00880-6.

Becker, M., Trautwein, U., Lüdtke, O., Cortina, K. S., \& Baumert, J. (2006). Bildungsexpansion und kognitive Mobilisierung. In A. Hadjar \& R. Becker (Hrsg.), Die Bildungsexpansion: Erwartete und unerwartete Folgen (S. 63-89). Wiesbaden: VS.

Becker, M., Lüdtke, O., Trautwein, U., Köller, O., \& Baumert, J. (2012). The differential effects of school tracking on psychometric intelligence: Do academic-track schools make students smarter? Journal of Educational Psychology, 104(3), 682-699. https://doi.org/10.1037/a0027608.

Becker, M., Neumann, M., \& Dumont, H. (2016). Recent developments in school tracking practices in Germany: an overview and outlook on future trends. Orbis Scholae, 10(3), 9-25. https://doi.org/10. 14712/23363177.2017.8.

Becker, M., Baumert, J., Tetzner, J., Maaz, K., \& Köller, O. (2019). Childhood intelligence, family background, and gender as drivers of socioeconomic success: the mediating role of education. Developmental Psychology. https://doi.org/10.1037/dev0000766.

Bol, T., \& van de Werfhorst, H. G. (2013). Educational systems and the trade-off between labor market allocation and equality of educational opportunity. Comparative Education Review, 57(2), 285-308. https://doi.org/10.1086/669122.

Brauckmann, S., \& Neumann, M. (2004). Berufliche Gymnasien in Baden-Württemberg: Geschichte und heutige Ausgestaltung. In O. Köller, R. Watermann, U. Trautwein \& O. Lüdtke (Hrsg.), Wege zur Hochschulreife in Baden-Württemberg. TOSCA - Eine Untersuchung an allgemeinbildenden und beruflichen Gymnasien (S. 69-111). Opladen: Leske + Budrich.

Buchholz, S., \& Pratter, M. (2017). Wer profitiert von alternativen Bildungswegen? Alles eine Frage des Blickwinkels! Eine systematische Rekonstruktion des Effektes sozialer Herkunft für alternative Wege zur Hochschulreife. Kölner Zeitschrift für Soziologie und Sozialpsychologie, 69, 409-435. https://doi. org/10.1007/s11577-017-0484-8. 
van Buuren, S., \& Groothuis-Oudshoorn, K. (2011). MICE: multivariate imputation by chained equations in R. Journal of Statistical Software, 45(3), 1-68. https://doi.org/10.18637/jss.v045.i03.

Chmielewski, A.K., \& Reardon, S.F. (2016). Patterns of cross-national variation in the association between income and academic achievement. AERA Open, 2(3), 1-27. https://doi.org/10.1177/ 2332858416649593.

Damian, R. I., Su, R., Shanahan, M., Trautwein, U., \& Roberts, B. W. (2015). Can personality traits and intelligence compensate for background disadvantage? Predicting status attainment in adulthood. Journal of Personality and Social Psychology, 109(3), 473-489. https://doi.org/10.1037/pspp0000024.

Ditton, H. (2010). Schullaufbahnen und soziale Herkunft - eine Frage von Leistung oder Diskriminierung? In S. Aufenanger, F. Hamburger, L. Ludwig \& R. Tippelt (Hrsg.), Bildung in der Demokratie (S. 79-99). Opladen: Barbara Budrich.

Dumont, H., Maaz, K., Neumann, M., \& Becker, M. (2014). Soziale Ungleichheiten beim Übergang von der Grundschule in die Sekundarstufe I: Theorie, Forschungsstand, Interventions- und Fördermöglichkeiten. In K. Maaz, M. Neumann \& J. Baumert (Hrsg.), Herkunft und Bildungserfolg von der frühen Kindheit bis ins Erwachsenenalter: Forschungsstand und Interventionsmöglichkeiten aus interdisziplinärer Perspektive (Zeitschrift für Erziehungswissenschaft: 24. Sonderheft, S. 141-165). Wiesbaden: Springer VS.

Dumont, H., Protsch, P., Jansen, M., \& Becker, M. (2017). Fish swimming into the ocean: How tracking relates to students' self-beliefs and school disengagement at the end of schooling. Journal of Educational Psychology, 109(6), 855-870. https://doi.org/10.1037/edu0000175.

Edelstein, W. (1970). Das „Projekt Schulleistung“ im Institut für Bildungsforschung in der Max-PlanckGesellschaft. Zeitschrift für Pädagogik, 16, 517-529.

Fend, H. (2009a). Arm und reich im frühen Erwachsenenalter: Wege zu Einkommen und Auskommen. In H. Fend, F. Berger \& U. Grob (Hrsg.), Lebensverläufe, Lebensbewältigung, Lebensglück: Ergebnisse der LifE-Studie (S. 161-192). Wiesbaden: VS.

Fend, H. (2009b). Chancengleichheit im Lebenslauf: Kurz- und Langzeitfolgen von Schulstrukturen. In H. Fend, F. Berger \& U. Grob (Hrsg.), Lebensverläufe, Lebensbewältigung, Lebensglück: Ergebnisse der LifE-Studie (S. 37-72). Wiesbaden: VS.

Fend, H. (2014). Bildungslaufbahnen von Generationen: Befunde der LifE-Studie zur Interaktion von Elternhaus und Schule. Zeitschrift für Erziehungswissenschaft, 17(1), 37-72. https://doi.org/10.1007/ s11618-013-0463-4.

Fend, H., Berger, F., \& Grob, U. (Hrsg.). (2009). Lebensverläufe, Lebensbewältigung, Lebensglück: Ergebnisse der LifE-Studie. Wiesbaden: VS.

Forster, A., Bol, T., \& Van de Werfhorst, H. G. (2016). Vocational education and employment over the lifecycle. Sociological Science, 3, 473-494. https://doi.org/10.15195/v3.a21.

Ganzach, Y. (2011). A dynamic analysis of the effects of intelligence and socioeconomic background on job-market success. Intelligence, 39, 120-129. https://doi.org/10.1016/j.intell.2011.02.003.

Ganzeboom, H. B. G. (2010). Occupational status measures for the new international standard classification of occupations isco-08; with a discussion of the new classification. http://www.harryganzeboom.nl/ isol/isol2010c2-ganzeboom.pdf. Zugegriffen: 21. Sept. 2020.

Ganzeboom, H. B. G., \& Treiman, D. J. (2003). Three internationally standardised measures for comparative research on occupational status. In J.H.P. Hoffmeyer-Zlotnik \& C. Wolf (Hrsg.), Advances in cross-national comparison: A European working book for demographic and socio-economic variables (S. 159-193). New York: Kluwer Academic Press.

Geis, A. (2011). Handbuch für die Berufsvercodung. https://www.gesis.org/fileadmin/upload/dienstleistung/ tools_standards/handbuch_der_berufscodierung_110304.pdf. Zugegriffen: 21. Sept. 2020.

Georg, W. (2009). Prädiktion des Berufsstatus: Zur unterschiedlichen Bedeutung personaler Ressourcen bei Frauen und Männern [Prediction of occupational status: on the differential importance of personal ressources in women and men. In H. Fend, F. Berger \& U. Grob (Hrsg.), Lebensverläufe, Lebensbewältigung, Lebensglück: Ergebnisse der LifE-Studie (S. 141-159). Wiesbaden: VS.

Graham, J. W. (2009). Missing data analysis: making it work in the real world. Annual Review in Psychology, 60, 549-576. https://doi.org/10.1146/annurev.psych.58.110405.085530.

Grund, S., Robitzsch, A., \& Lüdtke, O. (2016). mitml: Tools for multiple imputation in multilevel modeling: R package version 0.3-6, 2018. https://cran.r-project.org/web/packages/mitml/mitml.pdf. Zugegriffen: 21. Sept. 2020.

Hanushek, E. A., Schwerdt, G., Wiederhold, S., \& Woessmann, L. (2015). Returns to skills around the world: evidence from PIAAC. European Economic Review, 73, 103-130. 
Heckman, J. J., Lochner, L. J., \& Todd, P.E. (2006). Earnings functions, rates of return and treatment effects: the Mincer equation and beyond. In E. A. Hanushek \& F. Welch (Hrsg.), Handbook of the Economics of Education (Bd. 1, S. 307-458). Amsterdam: Elsevier.

Heller, K. A., \& Perleth, C. (2000). Kognitiver Fähigkeitstest für 4.-12. Klassen, Revision (KFT 4-12+R). Göttingen: Hogrefe.

Heller, K. A., Schoen-Gaedike, A.-K., \& Weinlaeder, H. (1985). Kognitiver Fähigkeitstest : KFT 4-13 (2. Aufl.). Weinheim: Beltz.

Hillmert, S. (2014). Bildung, Ausbildung und soziale Ungleichheiten im Lebenslauf. Zeitschrift für Erziehungswissenschaft, 17(1), 73-94. https://doi.org/10.1007/s11618-013-0465-2.

Hillmert, S., \& Jacob, M. (2005). Institutionelle Strukturierung und inter-individuelle Variation: Zur Entwicklung herkunftsbezogener Ungleichheiten im Bildungsverlauf. Kölner Zeitschrift für Soziologie und Sozialpsychologie, 57, 414-442. https://doi.org/10.1007/s11577-005-0183-8.

Hu, L.-T., \& Bentler, P. M. (1999). Cutoff criteria for fit indexes in covariance structure analysis: conventional criteria versus new alternatives. Structural Equation Modeling, 6(1), 1-55. https://doi.org/10. 1080/10705519909540118.

Hurrelmann, K. (2013). Das Schulsystem in Deutschland: Das „Zwei-Wege-Modell“ setzt sich durch. Zeitschrift für Pädagogik, 59(4), 455-468.

Husén, T. (1967). International study of achievement in mathematics: A comparison of 12 countries (Bd. 1,2). Stockholm: Almqvist \& Wiksell.

International Labor Office (Hrsg.). (1968). International Standard Classification of Occupations: Revised edition 1968. Genf: International Labour Office.

International Labor Office (2012). International Standard Classification of Occupations: ISCO-08: Vol. 1. Structure, group definitions and corresponcence tables. http://www.ilo.org/wcmsp5/groups/public/--dgreports/---dcomm/---publ/documents/publication/wcms_172572.pdf. Zugegriffen: 21. Sept. 2020.

International Labour Organization (1990). International standard classification of occupations: ISCO-88. Genf: ILO-International Labour Organization.

Kleinert, C., \& Jacob, M. (2012). Strukturwandel des Übergangs in eine berufliche Ausbildung. In R. Becker \& H. Solga (Hrsg.), Soziologische Bildungsforschung (Kölner Zeitschrift für Soziologie und Sozialpsychologie: Sonderheft 52, S. 211-233). Wiesbaden: Springer VS. https://doi.org/10.1007/ 978-3-658-00120-9_9.

Knigge, M., \& Hannover, B. (2011). Collective school type identity. Predicting students' motivation beyond academic self-concept. International Journal of Psychology, 46(3), 191-205. https://doi.org/ 10.1080/00207594.2010.529907.

Köller, O., Baumert, J., Cortina, K. S., Trautwein, U., \& Watermann, R. (2004a). Öffnung von Bildungswegen in der Sekundarstufe II und die Wahrung von Standards Analysen am Beispiel der Englischleistungen von Oberstufenschülern an integrierten Gesamtschulen, beruflichen und allgemein bildenden Gymnasien. Zeitschrift für Pädagogik, 50(5), 679-700.

Köller, O., Watermann, R., Trautwein, U., \& Lüdtke, O. (2004b). Wege zur Hochschulreife in BadenWürttemberg: TOSCA - Eine Untersuchung an allgemein bildenden und beruflichen Gymnasien. Opladen: Leske + Budrich.

Kuppens, T., Spears, R., Manstead, A.S., Spruyt, B., \& Easterbrook, M. J. (2017). Educationism and the irony of meritocracy: negative attitudes of higher educated people towards the less educated. Journal of Experimental Social Psychology, 76, 429-447. https://doi.org/10.1016/j.jesp.2017.11.001.

Leschinsky, A. (2003). Die Hauptschule - Sorgenkind im Schulwesen. In K. S. Cortina, J. Baumert, A. Leschinsky, K. U. Mayer \& L. Trommer (Hrsg.), Das Bildungswesen in der Bundesrepublik Deutschland: Strukturen und Entwicklungen im Überblick (S. 392-428). Reinbek bei Hamburg: Rowohlt.

Leschinsky, A. (2008). Die Realschule - ein zweischneidiger Erfolg. In K. S. Cortina, J. Baumert, A. Leschinsky, K. U. Mayer \& L. Trommer (Hrsg.), Das Bildungswesen in der Bundesrepublik Deutschland: Strukturen und Entwicklungen im Überblick (S. 407-435). Reinbek bei Hamburg: Rowohlt.

Liepmann, D., Beauducel, A., Brocke, B., \& Amthauer, R. (2007). I-S-T 2000 R: Intelligenz-Struktur-Test $2000 R$ (2. Aufl.). Göttingen: Hogrefe.

Little, R. J. A., \& Rubin, D. B. (2002). Statistical analysis with missing data (2. Aufl.). New York: Wiley.

Lucas, S.R. (2001). Effectively maintained inequality: Education transitions, track mobility, and social background effects. American Journal of Sociology, 106(6), 1642-1690.

Lucas, S. R. (2009). Stratification theory, socioeconomic background, and educational attainment: a formal analysis. Rationality and Society, 21, 459-511. https://doi.org/10.1177/1043463109348987.

Lüdtke, O., \& Robitzsch, A. (2011). Umgang mit fehlenden Daten in der empirischen Bildungsforschung. In S. Maschke \& L. Stecher (Hrsg.), Enzyklopädie Erziehungswissenschaft Online: Fachgebiet: Me- 
thoden der empirischen erziehungswissenschaftlichen Forschung, Quantitative Forschungsmethoden. Weinheim: Juventa. https://doi.org/10.3262/EEO07100150.

Maaz, K., Trautwein, U., Lüdtke, O., \& Baumert, J. (2008). Educational transitions and differential learning environments: how explicit between-school tracking contributes to social inequality in educational outcomes. Child Development Perspectives, 2, 99-106. https://doi.org/10.1111/j.1750-8606. 2008.00048.x.

Maaz, K., Baumert, J., Neumann, M., Becker, M., \& Dumont, H. (2013). Die Berliner Schulstrukturreform: Bewertung durch die beteiligten Akteure und Auswirkungen des neuen Übergangsverfahrens von der Grundschule in die weiterführende Schule. Münster: Waxmann.

Marsh, H. W., Seaton, M., Trautwein, U., Lüdtke, O., Hau, K. T., O’Mara, A. J., \& Craven, R. G. (2008). The big-fish-little-pond-effect stands up to critical scrutiny: implications for theory, methodology, and future research. Educational Psychology Review, 20(3), 319-350. https://doi.org/10.1007/s10648008-9075-6.

Mood, C. (2010). Logistic regression: why we cannot do what we think we can do, and what we can do about it. European Sociological Review, 26(1), 67-82. https://doi.org/10.1093/esr/jcp006.

Müller, W. (1998). Erwartete und unerwartete Folgen der Bildungsexpansion. In J. Friedrichs (Hrsg.), Die Diagnosefähigkeit der Soziologie (S. 81-112). Opladen: Westdeutscher Verlag.

Muthén, B. O., \& Muthén, L. K. (2013). Mplus (Version 7.11). Los Angeles: Computer software.

Neumann, M., Maaz, K., \& Becker, M. (2013). Die Abkehr von der traditionellen Dreigliedrigkeit im Sekundarschulsystem: Auf unterschiedlichen Wegen zum gleichen Ziel? Recht der Jugend und der Bildung, 61, 274-292.

Neumann, M., Baumert, J., Radmann, S., Becker, M., Maaz, K., \& Ohl, S. (2017a). Leistungs- und Bewertungsstandards beim Erwerb der Übergangsberechtigung in die gymnasiale Oberstufe im neu strukturierten Berliner Sekundarschulsystem. In M. Neumann, M. Becker, J. Baumert, K. Maaz \& O. Köller (Hrsg.), Zweigliedrigkeit im deutschen Schulsystem: Potenziale und Herausforderungen in Berlin (S. 255-294). Münster: Waxmann.

Neumann, M., Becker, M., Baumert, J., Maaz, K., \& Köller, O. (Hrsg.). (2017b). Zweigliedrigkeit im deutschen Schulsystem: Potenziale und Herausforderungen in Berlin. Münster: Waxmann.

Ordemann, J. (2019). Studium ohne Abitur: Bildungserträge nichttraditioneller Hochschulabsolventen im Vergleich. Wiesbaden: VS.

Protsch, P. (2014). Segmentierte Ausbildungsmärkte: Berufliche Chancen von Hauptschülerinnen und Hauptschülern im Wandel. Opladen: Budrich UniPress.

Psacharopoulos, G., \& Patrinos, H. A. (2004). Human capital and rates of return. In G. Johnes \& J. Johnes (Hrsg.), International handbook on the economics of edcuation. Cheltenham: Edward Elgar Publishing.

R Core Team (2019). R: A language and environment for statistical computing. Vienna, Austria: R Foundation for Statistical Computing. https://www.R-project.org/. Zugegriffen: 21. Sept. 2020.

Radmann, S., Neumann, M., Becker, M., \& Maaz, K. (2017). Leistungs- und lernerfahrungsbezogene Unterschiede zwischen promovierenden und nicht-promovierenden Hochschulabsolventen aus fachrichtungsübergreifender und fachrichtungsspezifischer Perspektive. Zeitschrift für Erziehungswissenschaft, 20(1), 113-138. https://doi.org/10.1007/s11618-016-0694-2.

Rubin, D.B. (1987). Multiple imputation for nonresponse in surveys. New York: Wiley.

Schermelleh-Engel, K., Moosbrugger, H., \& Müller, H. (2003). Evaluating the fit of structural equation models: tests of significance and descriptive goodness-of-fit measures. Methods of Psychological Research Online, 8(2), 23-74.

Schindler, S. (2014). Wege zur Studienberechtigung - Wege ins Studium? Eine Analyse sozialer Inklusionsund Ablenkungsprozesse. Wiesbaden: Springer VS.

Schoon, I. (2008). A transgenerational model of status attainment: The potential mediating role of school motivation and education. National Institute Economic Review, 205, 72-82. https://doi.org/10.1177/ 0027950108096590.

Schuchart, C., \& Rürup, M. (2017). Alternative Wege zur Studienberechtigung und die weitere Bildungsund Berufskarriere: Können durch die Öff nung des gegliederten Schulsystems Ungleichheiten reduziert werden? In T. Eckert \& B. Gniewosz (Hrsg.), Bildungsgerechtigkeit (S. 249-267). Wiesbaden: Springer. https://doi.org/10.1007/978-3-658-15003-7_15.

Schuchart, C., \& Schimke, B. (2019). Lohnt sich das Nachholen eines Schulabschlusses? Alternative Wege zur Hochschulreife und ihre Arbeitsmarkterträge. Kölner Zeitschrift für Soziologie und Sozialpsychologie, 71, 237-273. https://doi.org/10.1007/s11577-019-00621-x.

Settersten, R., \& Ray, B.E. (2010). Not quite adults: why 20-somethings are choosing a slower path to adulthood, and why it's good for everyone. New York: Random House. 
Solga, H., \& Wagner, S. (2001). Paradoxie der Bildungsexpansion: Die doppelte Benachteiligung von Hauptschülern. Zeitschrift für Erziehungswissenschaft, 4(1), 107-127. https://doi.org/10.1007/ s11618-001-0008-0.

Spence, M. (1973). Job market signaling. Quarterly Journal of Economics, 87(3), 355-374. https://doi.org/ $10.2307 / 1882010$.

Spengler, M., Brunner, M., Damian, R. I., Lüdtke, O., Martin, R., \& Roberts, B. W. (2015). Student characteristics and behaviors at age 12 predict occupational success 40 years later over and above childhood IQ and parental socioeconomic status. Developmental Psychology, 51(9), 1329-1340. https://doi.org/ $10.1037 / \mathrm{dev} 0000025$.

Spengler, M., Damian, R. I., \& Roberts, B. W. (2018). How you behave in school predicts life success above and beyond family background, broad traits, and cognitive ability. Journal of Personality and Social Psychology, 114, 620-636. https://doi.org/10.1037/pspp0000185.

Statistisches Bundesamt (1993). Bildung und Kultur (Fachserie 11, Reihe 1). Stuttgart: Metzler-Poeschel.

Sterrenberg, M. K. (2014). Chancengleichheit durch den Zweiten Bildungsweg? Langfristige Arbeitsmarkterträge später Hochschulreife (Diskussionsbeitrag Nr. 540). Hannover: Leibniz Universtität Hannover, Wirtschaftswissenschaftliche Fakultät.

Strenze, T. (2007). Intelligence and socioeconomic success: a meta-analytic review of longitudinal research. Intelligence, 35, 401-426. https://doi.org/10.1016/j.intell.2006.09.004.

von Stumm, S., Macintyre, S., Batty, D. G., Clark, H., \& Deary, I. J. (2010). Intelligence, social class of origin, childhood behavior disturbance and education as predictors of status attainment in midlife in men: the Aberdeen Children of the 1950s study. Intelligence, 38, 202-211. https://doi.org/10.1016/j. intell.2009.11.004.

Tetzner, J. (2014). Stability and change in academic, social, and emotional development from early adolescence to young adulthood: The interplay with negative life events and protective factors. Potsdam: Universität Potsdam.

Tillmann, K.-J. (2012). Das Sekundarschulsystem auf dem Weg in die Zweigliedrigkeit. Historische Linien und aktuelle Verwirrungen. Pädagogik, 64(5), 8-12.

Trautwein, U., Baerisyl, F., Lüdtke, O., \& Wandeler, C. (2008). Die Öffnung des Schulsystems: Fakt oder Fiktion? Empirische Befunde zum Zusammenhang von Grundschulübertritt und Übergang in die gymnasiale Oberstufe. Zeitschrift für Erziehungswissenschaft, 11(4), 648-665. https://doi.org/10. 1007/s11618-008-0043-1.

Trautwein, U., Nagy, G., \& Maaz, K. (2011). Soziale Disparitäten und die Öffnung des Sekundarschulsystems: Eine Studie zum Übergang von der Realschule in die gymnasiale Oberstufe. Zeitschrift für Erziehungswissenschaft, 14(3), 445-463. https://doi.org/10.1007/s11618-011-0220-5.

Travers, K., \& Westbury, I. (Hrsg.). (1989). The IEA study of mathematics (Bd. 1-3). Oxford: Pergamon Press.

Trautwein, U., Neumann, M., Nagy, G., Lüdtke, O., \& Maaz, K. (Hrsg.). (2010). Schulleistungen von Abiturienten: Die neu geordnete gymnasiale Oberstufe auf dem Prüfstand. Wiesbaden: VS.

Treiman, D. J. (1977). Occupational prestige in comparative perspective. New York: Academic Press.

Walker, D. (Hrsg.). (1976). IEA six subject survey: an empirical study of education in twenty-one countries. New York: Wiley.

Watermann, R., Daniel, A., \& Maaz, K. (2014). Primäre und sekundäre Disparitäten des Hochschulzugangs: Erklärungsmodelle, Datengrundlagen und Entwicklungen. Zeitschrift für Erziehungswissenschaft, 17(2), 233-261. https://doi.org/10.1007/s11618-013-0470-5.

Weiss, A. (1995). Human capital vs. signalling explanations of wages. Journal of Economic Perspectives, 9(4), 133-154. https://doi.org/10.1257/jep.9.4.133.

Weiss, F. (2013). Postsecondary educational careers and social inequality: An analysis of social origin differences in educational career trajectories and their labor market outcomes in the US, Sweden and Germany. (Doctoral thesis, Universität Mannheim, Germany). http://nbn-resolving.de/urn:nbn: de:0168-ssoar-384401. Zugegriffen: 21. Sept. 2020.

Wolter, A. (1989). Von der Elitenbildung zu Bildungsexpansion: 200 Jahre Abitur (1788-1988). Oldenburg: Bibliotheks- und Informationssystem der Universität Oldenburg.

Zax, J.S., \& Rees, D.I. (2002). IQ, academic performance, environment, and earnings. The Review of Economics and Statistics, 84, 600-616. https://doi.org/10.1162/003465302760556440. 